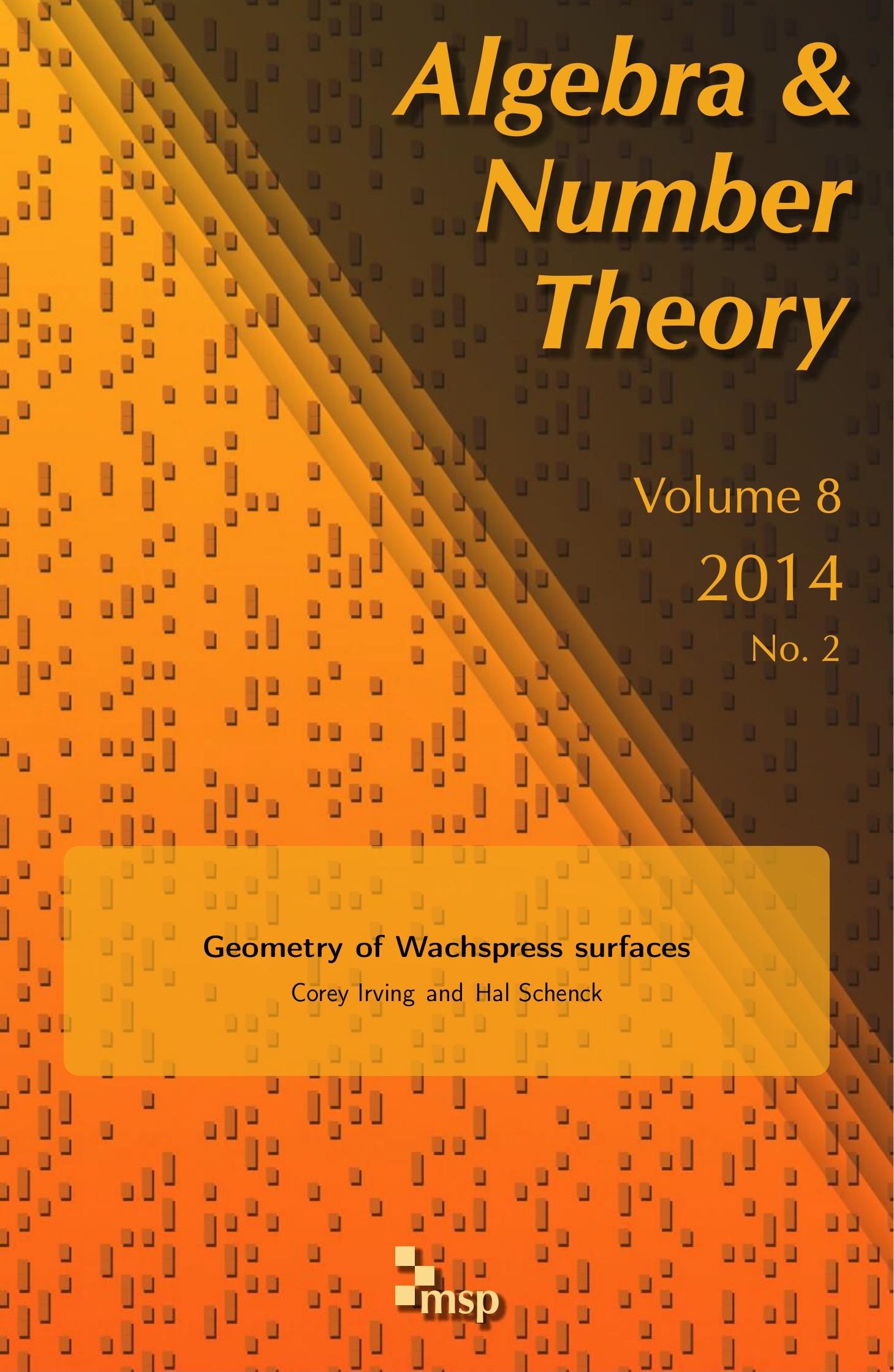




\title{
Geometry of Wachspress surfaces
}

\author{
Corey Irving and Hal Schenck
}

Let $P_{d}$ be a convex polygon with $d$ vertices. The associated Wachspress surface $W_{d}$ is a fundamental object in approximation theory, defined as the image of the rational map

$$
\mathbb{P}^{2} \stackrel{w_{d}}{\longrightarrow} \mathbb{P}^{d-1},
$$

determined by the Wachspress barycentric coordinates for $P_{d}$. We show $w_{d}$ is a regular map on a blowup $X_{d}$ of $\mathbb{P}^{2}$ and, if $d>4$, is given by a very ample divisor on $X_{d}$ so has a smooth image $W_{d}$. We determine generators for the ideal of $W_{d}$ and prove that, in graded lex order, the initial ideal of $I_{W_{d}}$ is given by a Stanley-Reisner ideal. As a consequence, we show that the associated surface is arithmetically Cohen-Macaulay and of Castelnuovo-Mumford regularity 2 and determine all the graded Betti numbers of $I_{W_{d}}$.

\section{Introduction}

Introduced by Möbius [1827], barycentric coordinates for triangles appear in a host of applications. Recent work in approximation theory has shown that it is also useful to define barycentric coordinates for a convex polygon $P_{d}$ with $d \geq 4$ vertices (a $d$-gon). The idea is as follows. To deform a planar shape, first place the shape inside a control polygon. Then move the vertices of the control polygon, and use barycentric coordinates to extend this motion to the entire shape.

For a $d$-gon with $d \geq 4$, barycentric coordinates were defined by Wachspress [1975] in his work on finite elements; these coordinates are rational functions depending on the vertices $v\left(P_{d}\right)$ of $P_{d}$. Warren [2003] shows that Wachspress' coordinates are the unique rational barycentric coordinates of minimal degree. The Wachspress coordinates define a rational map $w_{d}$ on $\mathbb{P}^{2}$, whose value at a point $p \in P_{d}$ is the $d$-tuple of barycentric coordinates of $p$. The closure of the image of $w_{d}$ is the Wachspress surface $W_{d}$, first defined and studied by Garcia-Puente and Sottile [2010] in their work on linear precision.

Irving is supported by Texas Advanced Research Program 010366-0054-2007. Schenck is supported by NSF 1068754 and NSA H98230-11-1-0170.

MSC2010: primary 13D02; secondary 52C35, 14J26, 14C20.

Keywords: barycentric coordinates, Wachspress variety, rational surface. 
In Definition 1.3, we fix linear forms $\ell_{i}$ that are positive inside $P_{d}$ and vanish on an edge. Let $A=\ell_{1} \cdots \ell_{d}, Z$ be the $\left(\begin{array}{l}d \\ 2\end{array}\right)$ singular points of $\mathbb{V}(A)$, and $Y=Z \backslash v\left(P_{d}\right)$. We call $Y$ the external vertices of $P_{d}$ and show that $w_{d}$ has basepoints only at $Y$. Let $X_{d}$ be the blowup of $\mathbb{P}^{2}$ at $Y$. In Section 2, we prove that $W_{d}$ is the image of $X_{d}$, embedded by a certain divisor $D_{d-2}$ on $X_{d}$. The global sections of $D_{d-2}$ have a simple interpretation in terms of the edges $\mathbb{V}\left(\ell_{i}\right)$ of $P_{d}$ : we prove that

$$
H^{0}\left(\mathcal{O}_{X_{d}}\left(D_{d-2}\right)\right) \text { has basis }\left\{\ell_{3} \cdots \ell_{d}, \ell_{1} \ell_{4} \cdots \ell_{d}, \ldots, \ell_{2} \cdots \ell_{d-1}\right\} .
$$

We show that $D_{d-2}$ is very ample if $d>4$; hence, $W_{d} \subseteq \mathbb{P}^{d-1}$ is a smooth surface.

1A. Statement of main results. For a $d$-gon $P_{d}$ with $d \geq 4$ :

(1) We give explicit generators for $I_{W_{d}} \subseteq S=\mathbb{K}\left[x_{1}, \ldots, x_{d}\right]$.

(2) We determine $\operatorname{in}_{\prec}\left(I_{W_{d}}\right)$, where $\prec$ is graded lex order.

(3) We prove in ${ }_{\prec}\left(I_{W_{d}}\right)$ is the Stanley-Reisner ideal of a graph $\Gamma$.

(4) We prove that $S / I_{W_{d}}$ is Cohen-Macaulay, and $\operatorname{reg}\left(S / I_{W_{d}}\right)=2$.

(5) We determine the graded Betti numbers of $S / I_{W_{d}}$.

In Section 1B, we give some quick background on geometric modeling, and in Section 1C, we do the same for algebraic geometry (in particular, we define all the terms above). Our strategy runs as follows. In Section 2, we study $I_{W_{d}}$ by blowing up $\mathbb{P}^{2}$ at the external vertices. Define a divisor

$$
D_{d-2}=(d-2) E_{0}-\sum_{p \in Y} E_{p}
$$

on $X_{d}$, where $E_{0}$ is the pullback of a line and $E_{p}$ is the exceptional fiber over $p$. We show that $D_{d-2}$ is very ample and that $I_{W_{d}}$ is the ideal of the image of

$$
X_{d} \rightarrow \mathbb{P}\left(H^{0}\left(D_{d-2}\right)\right) .
$$

Riemann-Roch then yields the Hilbert polynomial of $S / I_{W_{d}}$.

In Sections 3 and 4, we find distinguished sets of quadrics and cubics vanishing on $W_{d}$ and use them to generate a subideal $I(d) \subseteq I_{W_{d}}$. In Section 5, we tie everything together, showing that, in graded lex order, $I_{\Gamma}(d) \subseteq$ in $_{\prec} I(d)$, where $I_{\Gamma}(d)$ is the Stanley-Reisner ideal of a certain graph. Using results on flat deformations and an analysis of associated primes, we prove

$$
I_{\Gamma}(d)=\operatorname{in}_{\prec}(I(d)) .
$$

The description in terms of the Stanley-Reisner ring yields the Hilbert series for $S / I_{\Gamma}(d)$. We prove that $S / I_{\Gamma}(d)$ is Cohen-Macaulay and has CastelnuovoMumford regularity 2, and it follows from uppersemicontinuity that the same is true for $S / I(d)$. The differentials on the quadratic generators of $I_{\Gamma}(d)$ turn out to 
be easy to describe, and combining this with the regularity bound and knowledge of the Hilbert series yields the graded Betti numbers for in in $_{\prec}(I(d))$.

Finally, we show that $I(d)$ has no linear syzygies on its quadratic generators, which allows us to prune the resolution of $\operatorname{in}_{\prec}(I(d))$ to obtain the graded Betti numbers of $I(d)$. Comparing Hilbert polynomials shows that up to saturation

$$
S / I(d)=S / I_{W_{d}} .
$$

Since $I_{W_{d}}$ is prime, it is saturated, and a short-exact-sequence argument shows that $S / I(d)$ is also saturated, concluding the proof.

1B. Geometric modeling background. Let $P_{d}$ be a $d$-gon with vertices $v_{1}, \ldots, v_{d}$ and indices taken modulo $d$.

Definition 1.1. Functions $\left\{\beta_{i}: P_{d} \rightarrow \mathbb{R} \mid 1 \leq i \leq d\right\}$ are barycentric coordinates if, for all $p \in P_{d}$,

$$
\beta_{i}(p) \geq 0, \quad p=\sum_{i=1}^{d} \beta_{i}(p) v_{i}, \quad \sum_{i=1}^{d} \beta_{i}(p)=1 .
$$

Wachspress coordinates have a geometric description in terms of areas of subtriangles of the polygon. Let $A(a, b, c)$ denote the area of the triangle with vertices $a$, $b$, and $c$. For $1 \leq j \leq d$, set $\alpha_{j}:=A\left(v_{j-1}, v_{j}, v_{j+1}\right)$ and $A_{j}:=A\left(p, v_{j}, v_{j+1}\right)$.

Definition 1.2. For $1 \leq i \leq d$, the functions

$$
\beta_{i}=\frac{b_{i}}{\sum_{j=1}^{d} b_{j}}, \quad \text { where } b_{i}=\alpha_{i} \prod_{j \neq i-1, i} A_{j}
$$

are Wachspress barycentric coordinates for the $d$-gon $P_{d}$; see Figure 1 .

We embed $P_{d}$ in the plane $z=1 \subseteq \mathbb{R}^{3}$ and form the cone with $\mathbf{0} \in \mathbb{R}^{3}$. Explicitly, to each vertex $v_{i} \in v\left(P_{d}\right)$, we associate the ray $\boldsymbol{v}_{i}:=\left(v_{i}, 1\right) \in \mathbb{R}^{3}$. Let $\boldsymbol{P}_{d}$ denote the cone generated by the rays $\boldsymbol{v}_{i}$, and $v\left(\boldsymbol{P}_{d}\right):=\left\{\boldsymbol{v}_{i} \mid v_{i} \in v\left(P_{d}\right)\right\}$. The cone over

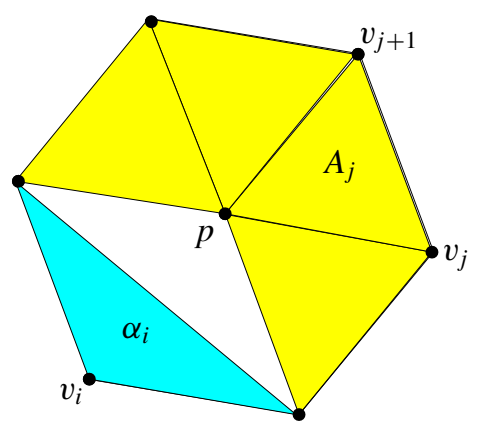

Figure 1. Wachspress coordinates for a polygon. 
the edge $\left[v_{i}, v_{i+1}\right]$ corresponds to a facet of $\boldsymbol{P}_{d}$ with normal vector $\boldsymbol{n}_{i}:=\boldsymbol{v}_{i} \times \boldsymbol{v}_{i+1}$. We redefine $\alpha_{j}$ and $A_{j}$ to be the determinants $\left|\boldsymbol{v}_{j-1} \boldsymbol{v}_{j} \boldsymbol{v}_{j+1}\right|$ and $\left|\boldsymbol{v}_{j} \boldsymbol{v}_{j+1} \boldsymbol{p}\right|$, where $\boldsymbol{p}=(x, y, z)$. This scales the $b_{i}$ by a factor of 2 so leaves the $\beta_{i}$ unchanged, save for homogenizing the $A_{j}$ with respect to $z$, and allows us to define Wachspress coordinates for nonconvex polygons, although Property 1 of barycentric coordinates fails when $P_{d}$ is nonconvex.

\section{Definition 1.3. $\quad \ell_{j}:=A_{j}=\boldsymbol{n}_{j} \cdot \boldsymbol{p}=\left|\boldsymbol{v}_{j} \boldsymbol{v}_{j+1} \boldsymbol{p}\right|$.}

The $\ell_{j}$ are homogeneous linear forms in $(x, y, z)$ and vanish on the cone over the edge $\left[v_{j}, v_{j+1}\right]$. We use Theorem 1.6 below, but Warren's proof does not require convexity. Our results hold over an arbitrary field $\mathbb{K}$ as long as no three of the lines $\mathbb{V}\left(\ell_{i}\right) \subseteq \mathbb{P}^{2}$ meet at a point. For the first condition of Definition 1.1 to make sense, $\mathbb{K}$ should be an ordered field.

Definition 1.4. The dual cone to $\boldsymbol{P}_{d}$ is the cone spanned by the normals $\boldsymbol{n}_{1}, \ldots, \boldsymbol{n}_{d}$ and is denoted $\boldsymbol{P}_{d}^{*}$.

Triangulating $P_{d}$ yields a triangulation of $\boldsymbol{P}_{d}$, and the volume of the parallelepiped $S$ spanned by vertices $\left\{\boldsymbol{v}_{i}, \boldsymbol{v}_{j}, \boldsymbol{v}_{k}, \mathbf{0}\right\}$ is $a_{S}=\left|\boldsymbol{v}_{i} \boldsymbol{v}_{j} \boldsymbol{v}_{k}\right|$.

Definition 1.5. Let $C$ be a cone defined by a polygon $P_{d}$ and $T(C)$ a triangulation of $C$ obtained from a triangulation of $P_{d}$ as above. The adjoint of $C$ is

$$
\mathscr{A}_{T(C)}(\boldsymbol{p})=\sum_{S \in T(C)} a_{S} \prod_{\boldsymbol{v} \in v\left(\boldsymbol{P}_{d}\right) \backslash v(S)}(\boldsymbol{v} \cdot \boldsymbol{p}) \in \mathbb{K}[x, y, z]_{d-3} .
$$

Theorem 1.6 [Warren 1996]. $\mathscr{A}_{T(C)}(\boldsymbol{p})$ is independent of the triangulation $T(C)$.

1C. Algebraic geometry background. Next, we review some background in algebraic geometry, referring to [Eisenbud 1995; Hartshorne 1977; Schenck 2003] for more detail. Homogenizing the numerators of Wachspress coordinates yields our main object of study:

Definition 1.7. The Wachspress map defined by a polygon $P_{d}$ is the rational map $\mathbb{P}^{2} \stackrel{w_{d}}{-\rightarrow} \mathbb{P}^{d-1}$ given on the open set $U_{z \neq 0} \subseteq \mathbb{P}^{2}$ by $(x, y) \mapsto\left(b_{1}(x, y), \ldots, b_{d}(x, y)\right)$. The Wachspress variety $W_{d}$ is the closure of the image of $w_{d}$.

The polynomial ring $S=\mathbb{K}\left[x_{1}, \ldots, x_{d}\right]$ is a graded ring: it has a direct-sum decomposition into homogeneous pieces. A finitely generated graded $S$-module $N$ admits a similar decomposition; if $s \in S_{p}$ and $n \in N_{q}$, then $s \cdot n \in N_{p+q}$. In particular, each $N_{q}$ is a $\left(S_{0}=\mathbb{K}\right)$-vector space.

Definition 1.8. For a finitely generated graded $S$-module $N$, the Hilbert series $\operatorname{HS}(N, t)=\sum \operatorname{dim}_{\mathbb{K}} N_{q} t^{q}$. 
Definition 1.9. A free resolution for an $S$-module $N$ is an exact sequence

$$
\mathbb{F}: \cdots \rightarrow F_{i} \stackrel{d_{i}}{\rightarrow} F_{i-1} \rightarrow \cdots \rightarrow F_{0} \rightarrow N \rightarrow 0,
$$

where the $F_{i}$ are free $S$-modules.

If $N$ is graded, then the $F_{i}$ are also graded, so letting $S(-m)$ denote a rank-1 free module generated in degree $m$, we may write $F_{i}=\bigoplus_{j} S(-j)^{a_{i, j}}$. By the Hilbert syzygy theorem [Eisenbud 1995], a finitely generated, graded $S$-module $N$ has a free resolution of length at most $d$ with all the $F_{i}$ of finite rank.

Definition 1.10. For a finitely generated graded $S$-module $N$, a free resolution is minimal if, for each $i, \operatorname{Im}\left(d_{i}\right) \subseteq \mathfrak{m} F_{i-1}$, where $\mathfrak{m}=\left\langle x_{1}, \ldots, x_{d}\right\rangle$. The graded Betti numbers of $N$ are the $a_{i, j}$ that appear in a minimal free resolution, and the Castelnuovo-Mumford regularity of $N$ is $\max _{i, j}\left\{a_{i, j}-i\right\}$.

While the differentials $d_{i}$ that appear in a minimal free resolution of $N$ are not unique, the ranks and degrees of the free modules that appear are unique. The graded Betti numbers are displayed in a Betti table. Reading this table right and down, starting at $(0,0)$, the entry $b_{i j}:=a_{i, i+j}$, and the regularity of $N$ is the index of the bottommost nonzero row in the Betti table for $N$.

Example 1.11. In Examples 2.9 and 3.11 of [Garcia-Puente and Sottile 2010], it is shown that $I_{W_{6}}$ is generated by three quadrics and one cubic. The variety $\mathbb{V}\left(\ell_{1} \cdots \ell_{6}\right)$ of the edges of $P_{6}$ has $\left(\begin{array}{l}6 \\ 2\end{array}\right)=15$ singular points, of which six are vertices of $P_{6}$, and $S / I_{W_{6}}$ has Betti table

\begin{tabular}{r|cccc} 
total & 1 & 4 & 6 & 3 \\
\hline 0 & 1 & - & - & - \\
1 & - & 3 & - & - \\
2 & - & 1 & 6 & 3
\end{tabular}

For example, $b_{1,2}=a_{1,3}=1$ reflects that $I_{W_{6}}$ has a cubic generator, and $S / I_{W_{6}}$ has regularity 2. The Hilbert series can be read off the Betti table:

$$
\operatorname{HS}\left(S / I_{W_{6}}, t\right)=\frac{1-3 t^{2}-t^{3}+6 t^{4}-3 t^{5}}{(1-t)^{6}}=\frac{1+3 t+3 t^{2}}{(1-t)^{3}} .
$$

Theorem 5.11 gives a complete description of the Betti table of $S / I_{W_{d}}$.

\section{2. $H^{0}\left(D_{d-2}\right)$ and the Wachspress surface}

2A. Background on blowups of $\mathbb{P}^{2}$. Fix points $p_{1}, \ldots, p_{k} \in \mathbb{P}^{2}$, and let

$$
X \stackrel{\pi}{\rightarrow} \mathbb{P}^{2}
$$

be the blowup of $\mathbb{P}^{2}$ at these points. Then $\operatorname{Pic}(X)$ is generated by the exceptional curves $E_{i}$ over the points $p_{i}$ and the proper transform $E_{0}$ of a line in $\mathbb{P}^{2}$. A classical 
geometric problem asks for a relationship between numerical properties of a divisor $D_{m}=m E_{0}-\sum a_{i} E_{i}$ on $X$ and the geometry of

$$
X \stackrel{\phi}{\rightarrow} \mathbb{P}\left(H^{0}\left(D_{m}\right)^{\vee}\right) .
$$

First, we discuss some basics. Let $m$ and $a_{i}$ be nonnegative, let $I_{p_{i}}$ denote the ideal of a point $p_{i}$, and define

$$
J=\bigcap_{i=1}^{k} I_{p_{i}}^{a_{i}} \subseteq \mathbb{K}[x, y, z]=R .
$$

Then $H^{0}\left(D_{m}\right)$ is isomorphic to the $m$-th graded piece $J_{m}$ of $J$ (see [Harbourne 2002]). Davis and Geramita [1988] show that, if $\gamma(J)$ denotes the smallest degree $t$ such that $J_{t}$ defines $J$ scheme theoretically, then $D_{m}$ is very ample if $m>\gamma(J)$, and if $m=\gamma(J)$, then $D_{m}$ is very ample if and only if $J$ does not contain $m$ collinear points, counted with multiplicity. Note that $\gamma(J) \leq \operatorname{reg}(J)$.

2B. Wachspress surfaces. For a polygon $P_{d}$, fix defining linear forms $\ell_{i}$ as in Definition 1.3 and let $A:=\ell_{1} \cdots \ell_{d}$; the edges of $P_{d}$ are defined by the $\mathbb{V}\left(\ell_{i}\right)$. Let $Z$ denote the $\left(\begin{array}{l}d \\ 2\end{array}\right)$ singular points of $\mathbb{V}(A)$ and $Y=Z \backslash v\left(P_{d}\right)$. Finally, $X_{d}$ will be the blowup of $\mathbb{P}^{2}$ at $Y$. We study the divisor

$$
D_{d-2}=(d-2) E_{0}-\sum_{p \in Y} E_{p}
$$

on $X_{d}$. First, we present some preliminaries.

Definition 2.1. Let $L$ be the ideal in $R=\mathbb{K}[x, y, z]$ given by

$$
L=\left\langle\ell_{3} \cdots \ell_{d}, \ell_{1} \ell_{4} \cdots \ell_{d}, \ldots, \ell_{2} \cdots \ell_{d-1}\right\rangle=\left\langle A / \ell_{1} \ell_{2}, A / \ell_{2} \ell_{3}, \ldots, A / \ell_{d} \ell_{1}\right\rangle,
$$

where $A=\prod_{i=1}^{d} \ell_{i}$.

For any variety $V$, we use $I_{V}$ to denote the ideal of polynomials vanishing on $V$.

Lemma 2.2. The ideals $L$ and $I_{Y}$ are equal up to saturation at $\langle x, y, z\rangle$.

Proof. Being equal up to saturation at $\langle x, y, z\rangle$ means that the localizations at any associated prime except $\langle x, y, z\rangle$ are equal. The ideal $I_{p}$ of a point $p$ is a prime ideal. Recall that the localization of a ring $T$ at a prime ideal $\mathfrak{p}$ is a new ring $T_{p}$ whose elements are of the form $f / g$ with $f, g \in T$ and $g \notin \mathfrak{p}$. Localize $R$ at $I_{p}$, where $p \in Y$. Then in $R_{I_{p}}, \ell_{i}$ is a unit if $p \notin \mathbb{V}\left(\ell_{i}\right)$. Without loss of generality, suppose forms $\ell_{1}$ and $\ell_{2}$ vanish on $p$ (note that all points of $Y$ are intersections of exactly two lines) and the remaining forms do not. Thus, $L_{I_{p}}=\left\langle\ell_{1}, \ell_{2}\right\rangle=\left(I_{Y}\right)_{I_{p}}$.

The ideal $L$ is not saturated.

Lemma 2.3. $I_{Y}$ is generated by one form $F$ of degree $d-3$ and $d-3$ forms of degree $d-2$. Hence, a basis for $L_{d-2}$ consists of $F \cdot x, F \cdot y, F \cdot z$, and the $d-3$ forms. 
Proof. First, note that $I_{Y}$ cannot contain any form of degree $d-4$ since $Y$ contains $d$ sets of $d-3$ collinear points. So the smallest degree of a minimal generator for $I_{Y}$ is $d-3$. Since $Y$ consists of $\left(\begin{array}{c}d-1 \\ 2\end{array}\right)-1$ distinct points and the space of forms of degree $d-3$ has dimension $\left(\begin{array}{c}d-1 \\ 2\end{array}\right)$, there is at least one form $F$ of degree $d-3$ in $I_{Y}$. We claim that it is unique. To see this, first note that no $\ell_{i}$ can divide $F$ : by symmetry, if one $\ell_{i}$ divides $F$, they all must, which is impossible for degree reasons. Now suppose $G$ is a second form of degree $d-3$ in $I_{Y}$. Let $p \in v\left(P_{d}\right)$ and $\mathbb{V}\left(\ell_{i}\right)$ be a line corresponding to an edge containing $p . F(p)$ must be nonzero since if not $\mathbb{V}(F)$ would contain $d-2$ collinear points of $\mathbb{V}\left(\ell_{i}\right)$, forcing $\mathbb{V}(F)$ to contain $\mathbb{V}\left(\ell_{i}\right)$, a contradiction. This also holds for $G$. But in this case, $F(p) G-G(p) F$ is a polynomial of degree $d-3$ vanishing at $d-2$ collinear points, again a contradiction. So $F$ is unique (up to scaling), which shows that the Hilbert function satisfies

$$
\operatorname{HF}(R / L, d-3)=|Y|,
$$

so $\operatorname{HF}(R / L, t)=|Y|$ for all $t \geq d-3$ (see [Schenck 2003]). As the polynomials $A / \ell_{i} \ell_{i+1}$ are linearly independent and there are the correct number, $L_{d-2}$ must be the degree- $(d-2)$ component of $I_{Y}$.

Theorem 2.4. The minimal free resolution of $R / L$ is

$0 \rightarrow R(-d) \stackrel{d_{3}}{\rightarrow} R(-d+1)^{d} \stackrel{d_{2}}{\rightarrow} R(-d+2)^{d} \stackrel{\left[\begin{array}{llll}\frac{A}{\ell_{1} \ell_{2}} & \frac{A}{\ell_{2} \ell_{3}} & \cdots & \frac{A}{\ell_{d} \ell_{1}}\end{array}\right]}{\longrightarrow} R \rightarrow R / L \rightarrow 0$

$$
\text { where } d_{2}=\left[\begin{array}{ccccccc}
\ell_{1} & 0 & \cdots & \cdots & 0 & 0 & m_{1} \\
-\ell_{3} & \ell_{2} & 0 & \cdots & \vdots & \vdots & m_{2} \\
0 & -\ell_{4} & \ddots & \ddots & \vdots & \vdots & \vdots \\
\vdots & 0 & \ddots & \ddots & \ell_{d-2} & 0 & \vdots \\
\vdots & \vdots & \ddots & \ddots & -\ell_{d} & \ell_{d-1} & \vdots \\
0 & \cdots & \cdots & 0 & 0 & -\ell_{1} & m_{d}
\end{array}\right]
$$

and the $m_{i}$ are linear forms.

Proof. By Lemma 2.3, the generators of $I_{Y}$ are known. Since $I_{Y}$ is saturated, the Hilbert-Burch theorem implies that the free resolution of $R / I_{Y}$ has the form

$$
0 \rightarrow R(-d+1)^{d-3} \rightarrow R(-d+3) \oplus R(-d+2)^{d-3} \rightarrow R \rightarrow R / I_{Y} \rightarrow 0 .
$$

Writing $I_{Y}$ as $\left\langle f_{1}, \ldots, f_{d-3}, F\right\rangle$ and $L$ as $\left\langle f_{1}, \ldots, f_{d-3}, x F, y F, z F\right\rangle$, the task is to understand the syzygies on $L$ given the description above of the syzygies on $I_{Y}$. From the Hilbert-Burch resolution, any minimal syzygy on $I_{Y}$ is of the form

$$
\sum g_{i} f_{i}+q F=0
$$


where $g_{i}$ are linear and $q$ is a quadric (or zero). Since

$$
q F=g_{1} x F+g_{2} y F+g_{3} z F \quad \text { with } g_{i} \text { linear, }
$$

all $d-3$ syzygies on $I_{Y}$ lift to give linear syzygies on $L$. Furthermore, we obtain three linear syzygies on $\{x F, y F, z F\}$ from the three Koszul syzygies on $\{x, y, z\}$. It is clear from the construction that these $d$ linear syzygies are linearly independent. Since $\operatorname{HF}(R / L, d-1)=|Y|$, this means we have determined all the linear first syzygies. Furthermore, the three Koszul first syzygies on $\{x F, y F, z F\}$ generate a linear second syzygy, so the complex given above is a subcomplex of the minimal free resolution. A check shows that the Buchsbaum-Eisenbud criterion [1973] holds, so the complex above is actually exact and hence a free resolution. The differential $d_{2}$ above involves the canonical generators $A / \ell_{i} \ell_{i+1}$ rather than a set involving $\{x F, y F, z F\}$. Since the $d-1$ linear syzygies appearing in the first $d-1$ columns of $d_{2}$ are linearly independent, they agree up to a change of basis; the last column of $d_{2}$ is a vector of linear forms determined by the change of basis.

\section{Theorem 2.5.}

(i) $H^{0}\left(D_{d-2}\right) \simeq \operatorname{Span}_{\mathbb{K}}\left\{A / \ell_{1} \ell_{2}, A / \ell_{2} \ell_{3}, \ldots\right\}$.

(ii) $H^{1}\left(D_{d-2}\right)=0=H^{2}\left(D_{d-2}\right)$.

Proof. The remark following Equation (2) shows that $H^{0}\left(D_{d-2}\right) \simeq L_{d-2}$. Since $K=-3 E_{0}+\sum_{p \in Y} E_{p}$ (see [Hartshorne 1977]), by Serre duality,

$$
H^{2}\left(D_{d-2}\right) \simeq H^{0}\left((-d-1) E_{0}+\sum_{p \in Y} E_{p}\right),
$$

which is clearly zero. Using that $X_{d}$ is rational, it follows from Riemann-Roch that

$$
h^{0}\left(D_{d-2}\right)-h^{1}\left(D_{d-2}\right)=\frac{D_{d-2}^{2}-D_{d-2} \cdot K}{2}+1 .
$$

The intersection pairing on $X_{d}$ is given by $E_{i}^{2}=1$ if $i=0$ and -1 if $i \neq 0$, and

$$
E_{i} \cdot E_{j}=0 \quad \text { if } i \neq j .
$$

Thus,

$$
D_{d-2}^{2}=(d-2)^{2}-|Y| \text { and } \quad-D_{d-2} K=3(d-2)-|Y|,
$$

yielding

$$
h^{0}\left(D_{d-2}\right)-h^{1}\left(D_{d-2}\right)=\frac{d^{2}-d-2-2|Y|}{2}+1=d .
$$

Thus, $h^{0}\left(D_{d-2}\right)-h^{1}\left(D_{d-2}\right)=d$. Now apply the remark following Equation (2).

Corollary 2.6. If $d>4, D_{d-2}$ is very ample, so the image of $X_{d}$ in $\mathbb{P}^{d-1}$ is smooth. 
Proof. By Theorem 2.4, the ideal $L$ is $d-2$ regular. Furthermore, the set $Y$ contains $d$ sets of $d-3$ collinear points but no set of $d-2$ collinear points if $d>4$. The result follows from the Davis-Geramita criterion.

Theorem 2.7. $W_{4} \simeq \mathbb{P}^{1} \times \mathbb{P}^{1}$, and $X_{4} \rightarrow W_{4}$ is an isomorphism away from the $(-1)$ curve $E_{0}-E_{1}-E_{2}$, which is contracted to a smooth point.

Proof. The surface $X_{4}$ is $\mathbb{P}^{2}$ blown up at two points, which is toric, and isomorphic to $\mathbb{P}^{1} \times \mathbb{P}^{1}$ blown up at a point. By Proposition 6.12 of [Cox et al. 2011], $D_{2}$ is basepoint free. Since $D_{2}^{2}=2, W_{4}$ is an irreducible quadric surface in $\mathbb{P}^{3}$. As $D_{2} \cdot\left(E_{0}-E_{1}-E_{2}\right)=0$, the result follows.

Replacing $D_{d-2}$ with $t D_{d-2}$, a computation as in Equations (3) and (4) and Serre vanishing shows that the Hilbert polynomial $\operatorname{HP}\left(S / I_{W_{d}}, t\right)$ is equal to

$$
\frac{\left((d-2)^{2}-|Y|\right) t^{2}+(3(d-2)-|Y|) t}{2}+1=\frac{d^{2}-5 d+8}{4} t^{2}-\frac{d^{2}-9 d+12}{4} t+1 .
$$

\section{The Wachspress quadrics}

In this section, we construct a set of quadrics that vanish on $W_{d}$. These quadrics are polynomials that are expressed as a scalar product with a fixed vector $\tau$. The vector $\tau$ defines a linear projection $\mathbb{P}^{d-1} \rightarrow \rightarrow \mathbb{P}^{2}$, also denoted by $\tau$, given by

$$
\boldsymbol{x} \mapsto \sum_{i=1}^{d} x_{i} \boldsymbol{v}_{i},
$$

where $\boldsymbol{x}=\left[x_{1}: \cdots: x_{d}\right] \in \mathbb{P}^{d-1}$. By the second property of barycentric coordinates, the composition $\tau \circ w_{d}: \mathbb{P}^{2} \rightarrow \mathbb{P}^{2}$ is the identity map on $\mathbb{P}^{2}$. Since $\boldsymbol{v}_{i} \in \mathbb{K}^{3}$, the vector $\tau$ is a triple of linear forms $\left(\tau_{1}, \tau_{2}, \tau_{3}\right) \in S^{3}$. The linear subspace $\mathscr{C}$ of $\mathbb{P}^{d-1}$ where the projection is undefined is the center of projection, and $I_{\mathscr{C}}=\left\langle\tau_{1}, \tau_{2}, \tau_{3}\right\rangle$.

3A. Diagonal monomials. A diagonal monomial is a monomial $x_{i} x_{j} \in S_{2}$ such that $j \notin\{i-1, i, i+1\}$. We write $\mathscr{D}$ for the subspace of $S_{2}$ spanned by the diagonal monomials; identifying $x_{i}$ with the vertex $v_{i}$, a diagonal monomial is a diagonal in $P_{d}$; see Figure 2.

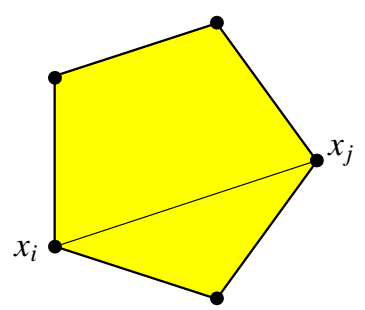

Figure 2. A diagonal monomial. 
Lemma 3.1. Any quadric that vanishes on $W_{d}$ is a linear combination of elements of D.

Proof. Let $Q$ be a polynomial in $\left(I_{W_{d}}\right)_{2}$. Then $Q\left(w_{d}\right)=Q\left(b_{1}, \ldots, b_{d}\right)=0$. On the edge $\left[v_{k}, v_{k+1}\right]$, all the $b_{i}$ vanish except $b_{k}$ and $b_{k+1}$. Thus, on this edge, the expression $Q\left(w_{d}\right)=0$ is

$$
c_{1} b_{k}^{2}+c_{2} b_{k} b_{k+1}+c_{3} b_{k+1}^{2}=0
$$

for some constants $c_{1}, c_{2}$, and $c_{3}$ in $\mathbb{K}$. Recall that $b_{i}\left(v_{j}\right)=0$ if $i \neq j$ and $b_{i}\left(v_{i}\right) \neq 0$ for each $i$. Evaluating (6) at $v_{k}$ and $v_{k+1}$, we conclude $c_{1}=c_{3}=0$. At an interior point of edge $\left[v_{k}, v_{k+1}\right]$, neither $b_{k}$ nor $b_{k+1}$ vanishes. This implies that $c_{2}=0$. A similar calculation on each edge shows that all coefficients of nondiagonal terms in $Q$ are zero.

3B. The map to $\left(I_{\mathscr{C}}\right)_{2}$. We define a surjective map onto $\left(I_{\mathscr{C}}\right)_{2}$ and use the map to calculate the dimension of the vector space of polynomials in $\left(I_{\mathscr{C}}\right)_{2}$ that are supported on diagonal monomials. Let $S_{1}^{3}$ denote the space of triples of linear forms on $\mathbb{P}^{d-1}$. Define the map $\Psi: S_{1}^{3} \rightarrow\left(I_{\mathscr{C}}\right)_{2}$ by $F \mapsto F \cdot \tau$, where $\cdot$ is the scalar product.

Lemma 3.2. The kernel of $\Psi$ is three-dimensional.

Proof. Since $I_{\mathscr{C}}$ is a complete intersection, the kernel is generated by the three Koszul syzygies on the $\tau_{i}$.

Next we determine conditions on $F$ so that $\Psi(F) \in \mathscr{D}$. If $\boldsymbol{u}_{i} \in \mathbb{K}^{3}$ for $i=1, \ldots, d$, then

$$
F=\sum_{i=1}^{d} x_{i} \boldsymbol{u}_{i}
$$

is an element of $S_{1}^{3}$. Viewing the projection $\tau$ as an element of $S_{1}^{3}$, we have

$$
\Psi(F)=F \cdot \tau=\left(\sum_{i=1}^{d} x_{i} \boldsymbol{u}_{i}\right) \cdot\left(\sum_{i=1}^{d} x_{i} \boldsymbol{v}_{i}\right)=\sum_{i, j=1}^{d}\left(\boldsymbol{u}_{i} \cdot \boldsymbol{v}_{j}+\boldsymbol{u}_{j} \cdot \boldsymbol{v}_{i}\right) x_{i} x_{j} .
$$

If $\Psi(F) \in \mathscr{D}$, then the coefficients of nondiagonal monomials must vanish:

$$
\boldsymbol{u}_{i} \cdot \boldsymbol{v}_{i}=0 \quad \text { and } \quad \boldsymbol{u}_{i} \cdot \boldsymbol{v}_{i+1}+\boldsymbol{u}_{i+1} \cdot \boldsymbol{v}_{i}=0 \quad \text { for all } i
$$

Lemma 3.3. The dimension of the vector space $\mathscr{D} \cap\left(I_{\mathscr{C}}\right)_{2}$ is $d-3$.

Proof. We show the conditions in (8) give $2 d$ independent conditions on the $3 d$-dimensional vector space $S_{1}^{3}$, and the solution space is $\Psi^{-1}\left(\mathscr{D} \cap\left(I_{\mathscr{C}}\right)_{2}\right)$; thus, 
$\operatorname{dim}\left(\Psi^{-1}\left(\mathscr{D} \cap\left(I_{\mathscr{C}}\right)_{2}\right)\right)=d$. The conditions are represented by the matrix equation

$$
\left(\begin{array}{c}
\boldsymbol{v}_{1} \cdot \boldsymbol{u}_{1} \\
\vdots \\
\boldsymbol{v}_{d} \cdot \boldsymbol{u}_{d} \\
\boldsymbol{v}_{1} \cdot \boldsymbol{u}_{2}+\boldsymbol{v}_{2} \cdot \boldsymbol{u}_{1} \\
\vdots \\
\boldsymbol{v}_{d} \cdot \boldsymbol{u}_{1}+\boldsymbol{v}_{1} \cdot \boldsymbol{u}_{d}
\end{array}\right)=\overbrace{\left(\begin{array}{cccc}
\boldsymbol{v}_{1}^{T} & 0 & \cdots & 0 \\
0 & \boldsymbol{v}_{2}^{T} & & \vdots \\
\vdots & & \ddots & 0 \\
0 & \cdots & 0 & \boldsymbol{v}_{d}^{T} \\
\boldsymbol{v}_{2}^{T} & \boldsymbol{v}_{1}^{T} & & 0 \\
0 & & \ddots & \\
\boldsymbol{v}_{d}^{T} & & & \boldsymbol{v}_{1}^{T}
\end{array}\right)}^{M}\left(\begin{array}{c}
\boldsymbol{u}_{1} \\
\boldsymbol{u}_{2} \\
\vdots \\
\vdots \\
\vdots \\
\vdots \\
\boldsymbol{u}_{d}
\end{array}\right)=\left(\begin{array}{c}
0 \\
0 \\
\vdots \\
\vdots \\
\vdots \\
0
\end{array}\right),
$$

where the $\boldsymbol{v}_{i}$ and $\boldsymbol{u}_{i}$ are column vectors and the superscript $T$ indicates transpose. The matrix $M$ in the middle is a $2 d \times 3 d$ matrix, and the proof will be complete if the rows are shown to be independent. Denote the rows of $M$ by $r_{1}, \ldots, r_{d}, r_{d+1}, \ldots, r_{2 d}$, and let $c_{1} r_{1}+\cdots+c_{d} r_{d}+c_{d+1} r_{d+1}+\cdots+c_{2 d} r_{2 d}$ be a dependence relation among them. The first three columns of $M$ give the dependence relation $c_{1} \boldsymbol{v}_{1}+c_{d+1} \boldsymbol{v}_{2}+c_{2 d} \boldsymbol{v}_{d}=0$. Since $\boldsymbol{v}_{d}, \boldsymbol{v}_{1}$, and $\boldsymbol{v}_{2}$ define adjacent rays of a polyhedral cone, they must be independent, so $c_{1}, c_{d+1}$, and $c_{2 d}$ must be zero. Repeating the process at each triple $\boldsymbol{v}_{i-1}, \boldsymbol{v}_{i}$, and $\boldsymbol{v}_{i+1}$ shows the rest of the $c_{i}$ 's vanish. Since the restriction $\Psi: \Psi^{-1}\left(\mathscr{D} \cap\left(I_{\mathscr{C}}\right)_{2}\right) \rightarrow \mathscr{D} \cap\left(I_{\mathscr{C}}\right)_{2}$ remains surjective, we find $\operatorname{dim}\left(\mathscr{D} \cap\left(I_{\mathscr{C}}\right)_{2}\right)=\operatorname{dim}\left(\Psi^{-1}\left(\mathscr{D} \cap\left(I_{\mathscr{C}}\right)_{2}\right)\right)-\operatorname{dim}(\operatorname{ker}(\Psi))=d-3$.

3C. Wachspress quadrics. We now compute the dimension and a generating set for $\left(I_{W_{d}}\right)_{2}$.

Definition 3.4. Let $\gamma(i)$ denote the set $\{1, \ldots, d\} \backslash\{i-1, i\}, \gamma(i, j)=\gamma(i) \cap \gamma(j)$, and $\gamma(i, j, k)=\gamma(i) \cap \gamma(j) \cap \gamma(k)$.

The image of a diagonal monomial $x_{i} x_{j}$ under the pullback map $w_{d}^{*}: S \rightarrow R$ is

$$
b_{i} b_{j}=\alpha_{i} \alpha_{j} \prod_{k \in \gamma(i)} \ell_{k} \prod_{m \in \gamma(j)} \ell_{m}=\alpha_{i} \alpha_{j} \prod_{k=1}^{d} \ell_{k} \prod_{m \in \gamma(i, j)} \ell_{m},
$$

and each diagonal monomial has a common factor $A=\prod_{k=1}^{d} \ell_{k}$. To find the quadratic relations among Wachspress coordinates, it suffices to find linear relations among products $\prod_{m \in \gamma(i, j)} \ell_{m} \in R_{d-4}$ for diagonal pairs $i$ and $j$. Define the map $\phi: \mathscr{D} \rightarrow R_{d-4}$ by $x_{i} x_{j} \mapsto b_{i} b_{j} / A$, and extend by linearity; this is $w_{d}^{*}$ restricted to $\mathscr{D}$ and divided by $A$. By Lemma 3.1, it follows that $\left(I_{W_{d}}\right)_{2}=\operatorname{ker}(\phi) \subseteq \mathscr{D}$.

Lemma 3.5. The dimension of $\left(I_{W_{d}}\right)_{2}$ is $d-3$.

Proof. We will show $\phi: \mathscr{D} \rightarrow R_{d-4}$ is surjective with $\operatorname{dim}(\operatorname{ker} \phi)=d-3$. To see this, note that there are $d-3$ diagonal monomials that have $x_{1}$ as a factor. We show 


$$
\begin{array}{llllllllll}
x_{2,4} & \cdots & x_{2, d} & x_{3,5} & \cdots & x_{3, d} & \cdots & x_{d-3, d-1} & x_{d-3, d} & x_{d-2, d}
\end{array}
$$

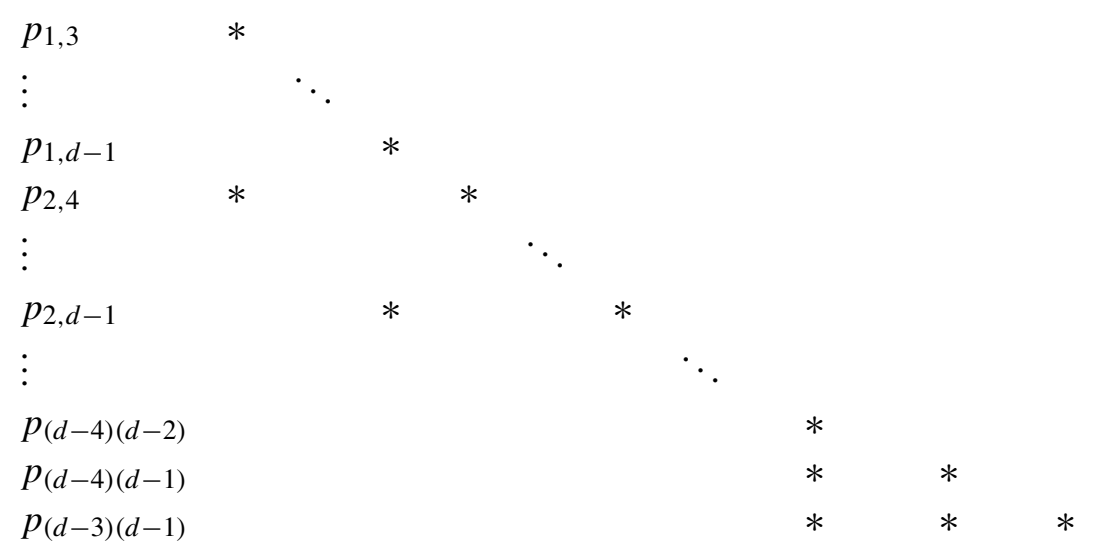

Table 1. Values of images of diagonal monomials at external vertices.

that the images of the remaining

$$
d(d-3) / 2-(d-3)=(d-3)(d-2) / 2=\operatorname{dim}\left(R_{d-4}\right)
$$

diagonal monomials are independent. Let $p_{s, t}=\ell_{s} \cap \ell_{t}$ and $x_{p, q}=x_{p} x_{q}$. In Table 1, a star, $*$, represents a nonzero number and a blank space is zero. The $(i, j)$ entry in the table represents the value of the image of the diagonal monomial in column $j$ at the external vertex in row $i$. The external vertices not lying on $\ell_{d}$ are arranged down the rows with their indices in lexicographic order.

Since Table 1 is lower triangular, the images are independent. We have found $\operatorname{dim}\left(R_{d-4}\right)$ independent images, and hence, $\phi$ is surjective. This is a map from a vector space of dimension $d(d-3) / 2$ to one of dimension $(d-2)(d-3) / 2$. The map is surjective, so the kernel has dimension $d(d-3) / 2-(d-2)(d-3) / 2=d-3$.

There is a generating set for $\left(I_{W_{d}}\right)_{2}$ where each generator is a scalar product with the vector $\tau$. The other vectors in these scalar products are

$$
\Lambda_{k}=\frac{x_{k+1}}{\alpha_{k+1}} \boldsymbol{n}_{k+1}-\frac{x_{k}}{\alpha_{k}} \boldsymbol{n}_{k-1} \in S_{1}^{3} .
$$

Lemma 3.6. The vectors $\left\{\Lambda_{1}, \ldots, \Lambda_{d}\right\}$ form a basis for the space $\Psi^{-1}\left(\mathscr{D} \cap\left(I_{\mathscr{C}}\right)_{2}\right)$. Proof. Suppose that $\sum_{k=1}^{d} c_{k} \Lambda_{k}=0$ is a linear dependence relation among the $\Lambda_{k}$. The coefficient of a variable $x_{k}$ is

$$
\frac{1}{\alpha_{k}}\left(c_{k-1} \boldsymbol{n}_{k}-c_{k} \boldsymbol{n}_{k-1}\right) \text {. }
$$

By the dependence relation, this must be zero, which implies that $\boldsymbol{n}_{k-1}$ and $\boldsymbol{n}_{k}$ are scalar multiples. This is impossible since they are normal vectors of adjacent facets 
of a polyhedral cone. Hence, $c_{k-1}=c_{k}=0$ for all $k$, which shows that the $\Lambda_{k}$ are independent.

In the proof of Lemma 3.3, we showed that $\operatorname{dim}\left(\Psi^{-1}\left(\mathscr{D} \cap\left(I_{\mathscr{C}}\right)_{2}\right)\right)=d$, and we have just shown $\operatorname{dim}\left(\left\langle\Lambda_{k} \mid k=1, \ldots, d\right\rangle\right)=d$. To prove the result, it suffices to show $\left\langle\Lambda_{k} \mid k=1, \ldots, d\right\rangle \subseteq \Psi^{-1}\left(\mathscr{D} \cap\left(I_{\mathscr{C}}\right)_{2}\right)$. The conditions of (8) are required for $\Lambda_{k} \in S_{1}^{3}$ to lie in $\Psi^{-1}\left(\mathscr{D} \cap\left(I_{\mathscr{C}}\right)_{2}\right)$. We show these conditions are satisfied for each $\Lambda_{k}$.

Let $\boldsymbol{u}_{i}=0$ if $i \neq k, k+1, \boldsymbol{u}_{k}=-\boldsymbol{n}_{k-1} / \alpha_{k}$, and $\boldsymbol{u}_{k+1}=\boldsymbol{n}_{k+1} / \alpha_{k+1}$ for each fixed $k$. Then

$$
\Lambda_{k}=\frac{x_{k+1}}{\alpha_{k+1}} \boldsymbol{n}_{k+1}-\frac{x_{k}}{\alpha_{k}} \boldsymbol{n}_{k-1}=\sum_{i=1}^{d} \boldsymbol{u}_{i} x_{i} .
$$

Since $\boldsymbol{n}_{k-1} \cdot \boldsymbol{v}_{k}=0, \boldsymbol{n}_{k+1} \cdot \boldsymbol{v}_{k+1}=0$, and $\boldsymbol{u}_{i}=0$ for $i \neq k, k+1$, we have that $\boldsymbol{u}_{i} \cdot \boldsymbol{v}_{i}=0$ for each $i=1, \ldots d$. The expression $\boldsymbol{u}_{i} \cdot \boldsymbol{v}_{i+1}+\boldsymbol{u}_{i+1} \cdot \boldsymbol{v}_{i}$ is zero for all $i \neq k-1, k, k+1$ simply because $\boldsymbol{u}_{i}=0$ for $i \neq k, k+1$. We have

$$
\begin{aligned}
\boldsymbol{u}_{k} \cdot \boldsymbol{v}_{k+1}+\boldsymbol{u}_{k+1} \cdot \boldsymbol{v}_{k} & =-\frac{\boldsymbol{n}_{k-1}}{\alpha_{k}} \cdot \boldsymbol{v}_{k+1}+\frac{\boldsymbol{n}_{k+1}}{\alpha_{k+1}} \cdot \boldsymbol{v}_{k} \\
& =-\frac{\boldsymbol{v}_{k-1} \times \boldsymbol{v}_{k} \cdot \boldsymbol{v}_{k+1}}{\alpha_{k}}+\frac{\boldsymbol{v}_{k+1} \times \boldsymbol{v}_{k+2} \cdot \boldsymbol{v}_{k}}{\alpha_{k+1}} \\
& =-\frac{\left|\boldsymbol{v}_{k-1} \boldsymbol{v}_{k} \boldsymbol{v}_{k+1}\right|}{\alpha_{k}}+\frac{\left|\boldsymbol{v}_{k+1} \boldsymbol{v}_{k+2} \boldsymbol{v}_{k}\right|}{\alpha_{k+1}}=0
\end{aligned}
$$

as $\alpha_{j}=\left|\boldsymbol{v}_{j-1} \boldsymbol{v}_{j} \boldsymbol{v}_{j+1}\right|$. It is easy to show that the expression $\boldsymbol{u}_{i} \cdot \boldsymbol{v}_{i+1}+\boldsymbol{u}_{i+1} \cdot \boldsymbol{v}_{i}$ is zero for $i=k \pm 1$. Thus, the $\boldsymbol{u}_{i}$ satisfy the conditions in (8), so $\Lambda_{k} \in \Psi^{-1}\left(\mathscr{D} \cap\left(I_{\mathscr{C}}\right)_{2}\right)$.

Theorem 3.7 (Wachspress quadrics). The Wachspress quadrics $\left(I_{W_{d}}\right)_{2}$ are those elements of $S_{2}$ that are diagonally supported and vanish on $\mathscr{C}$. The quadrics $Q_{k}=\Lambda_{k} \cdot \tau$ for $k=1, \ldots, d \operatorname{span}\left(I_{W_{d}}\right)_{2}$.

Proof. Let $\boldsymbol{p}$ be the vector $(x, y, z)$. By definition of Wachspress coordinates,

$$
\tau\left(w_{d}(\boldsymbol{p})\right)=\sum_{i=1}^{d} b_{i}(\boldsymbol{p}) \boldsymbol{v}_{i}=\boldsymbol{p} \sum_{i=1}^{d} b_{i}(\boldsymbol{p}) .
$$

We have

$$
\begin{aligned}
\Lambda_{k}\left(w_{d}(\boldsymbol{p})\right) & =\frac{b_{k+1}(\boldsymbol{p})}{\alpha_{k+1}} \boldsymbol{n}_{k+1}-\frac{b_{k}(\boldsymbol{p})}{\alpha_{k}} \boldsymbol{n}_{k-1} \\
& =\left(\prod_{j \neq k, k+1} \ell_{j}\right) \boldsymbol{n}_{k+1}-\left(\prod_{j \neq k-1, k} \ell_{j}\right) \boldsymbol{n}_{k-1} \\
& =\left(\prod_{j \neq k-1, k, k+1} \ell_{j}\right)\left(\ell_{k-1} \boldsymbol{n}_{k+1}-\ell_{k+1} \boldsymbol{n}_{k-1}\right) \\
& =H\left[\boldsymbol{n}_{k+1}\left(\boldsymbol{n}_{k-1} \cdot \boldsymbol{p}\right)-\boldsymbol{n}_{k-1}\left(\boldsymbol{n}_{k+1} \cdot \boldsymbol{p}\right)\right],
\end{aligned}
$$


where $H=\prod_{j \neq k-1, k, k+1} \ell_{j}$. Set $\bar{H}:=H \sum_{i=1}^{d} b_{i}(\boldsymbol{p})$. Then we have

$$
\begin{aligned}
Q_{k}\left(w_{d}(\boldsymbol{p})\right) & =\tau\left(w_{d}(\boldsymbol{p})\right) \cdot \Lambda_{k}\left(w_{d}(\boldsymbol{p})\right) \\
& =\bar{H} \boldsymbol{p} \cdot\left[\boldsymbol{n}_{k+1}\left(\boldsymbol{n}_{k-1} \cdot \boldsymbol{p}\right)-\boldsymbol{n}_{k-1}\left(\boldsymbol{n}_{k+1} \cdot \boldsymbol{p}\right)\right] \\
& =\bar{H}\left[\left(\boldsymbol{n}_{k+1} \cdot \boldsymbol{p}\right)\left(\boldsymbol{n}_{k-1} \cdot \boldsymbol{p}\right)-\left(\boldsymbol{n}_{k-1} \cdot \boldsymbol{p}\right)\left(\boldsymbol{n}_{k+1} \cdot \boldsymbol{p}\right)\right]=0 .
\end{aligned}
$$

We have just shown that $Q_{k} \in\left(I_{W_{d}}\right)_{2}$. By Lemma 3.6, $\Psi^{-1}\left(\mathscr{D} \cap\left(I_{\mathscr{C}}\right)_{2}\right)$ is spanned by the $\Lambda_{k}$. Observe that $\left\langle Q_{1}, \ldots, Q_{d}\right\rangle=\Psi\left(\left\langle\Lambda_{k}\right\rangle\right)=\mathscr{D} \cap\left(I_{\mathscr{C}}\right)_{2}$. Thus, $\operatorname{dim}\left(\left\langle Q_{1}, \ldots, Q_{d}\right\rangle\right)=d-3$, and by Lemma 3.5, $\operatorname{dim}\left(\left(I_{W_{d}}\right)_{2}\right)=d-3$. Therefore, since $\left\langle Q_{1}, \ldots, Q_{d}\right\rangle \subseteq\left(I_{W_{d}}\right)_{2}$, we have $\left\langle Q_{1}, \ldots, Q_{d}\right\rangle=\left(I_{W_{d}}\right)_{2}=\mathscr{D} \cap\left(I_{\mathscr{C}}\right)_{2}$.

Corollary 3.8. The quadrics $\left\{\Lambda_{2} \cdot \tau, \ldots, \Lambda_{d-2} \cdot \tau\right\}$ are a basis for the quadrics in $I_{W_{d}}$, and in graded lex order, $\left\{x_{1} x_{3}, \ldots, x_{1} x_{d-1}\right\}$ is a basis for $\operatorname{in}_{\prec}\left(I_{W_{d}}\right)_{2}$.

Proof. Expanding the expression for $\Lambda_{i} \cdot \tau$ yields

$$
\Lambda_{i} \cdot \tau=x_{1} x_{i+1}\left(\frac{\boldsymbol{v}_{1} \cdot \boldsymbol{n}_{i+1}}{\alpha_{i+1}}\right)-x_{1} x_{i}\left(\frac{\boldsymbol{v}_{1} \cdot \boldsymbol{n}_{i-1}}{\alpha_{i}}\right)+\zeta_{i},
$$

where $\zeta_{i} \in \mathbb{K}\left[x_{2}, \ldots, x_{d}\right]$. Since $\boldsymbol{n}_{i}=\boldsymbol{v}_{i} \times \boldsymbol{v}_{i+1}$,

$$
\Lambda_{2} \cdot \tau=x_{1} x_{3}\left(\frac{\boldsymbol{v}_{1} \cdot \boldsymbol{n}_{3}}{\alpha_{3}}\right)+\zeta_{2} \text {. }
$$

Since no three of the lines $\mathbb{V}\left(l_{i}\right)$ are concurrent, $\boldsymbol{v}_{i} \cdot \boldsymbol{n}_{j}$ is nonzero unless $j \in\{i, i+1\}$, so we may use the lead term of $\Lambda_{2} \cdot \tau$ to reduce $\Lambda_{3} \cdot \tau$ to $x_{1} x_{4}+f\left(x_{2}, \ldots, x_{d}\right)$. Repeating the process proves that

$$
\left\{x_{1} x_{3}, \ldots, x_{1} x_{d-1}\right\} \subseteq \operatorname{in}_{\prec}\left(I_{W_{d}}\right)_{2} .
$$

By Lemma 3.5, $\left(I_{W_{d}}\right)_{2}$ has dimension $d-3$, which concludes the proof.

Corollary 3.9. There are no linear first syzygies on $\left(I_{W_{d}}\right)_{2}$.

Proof. By Corollary 3.8, we may assume that a basis for $\left(I_{W_{d}}\right)_{2}$ has the form

$$
\begin{gathered}
x_{1} x_{3}+\zeta_{3}\left(x_{2}, \ldots, x_{d}\right), \\
x_{1} x_{4}+\zeta_{4}\left(x_{2}, \ldots, x_{d}\right), \\
x_{1} x_{5}+\zeta_{5}\left(x_{2}, \ldots, x_{d}\right), \\
\vdots \\
x_{1} x_{d-1}+\zeta_{d-1}\left(x_{2}, \ldots, x_{d}\right) .
\end{gathered}
$$

Since the $\zeta_{i}$ do not involve $x_{1}$, this implies that any linear first syzygy on $\left(I_{W_{d}}\right)_{2}$ must be a linear combination of the Koszul syzygies on $\left\{x_{3}, \ldots, x_{d-1}\right\}$. Now change the term order to graded lex with $x_{i}>x_{i+1}>\cdots>x_{d}>x_{1}>x_{2}>\cdots>x_{i-1}$. In 
this order, arguing as in the proof of Corollary 3.8 shows that we may assume a basis for $\left(I_{W_{d}}\right)_{2}$ has the form

$$
\begin{gathered}
x_{i} x_{i+2}+\zeta_{i+2}\left(x_{1}, \ldots, \widehat{x_{i}}, \ldots, x_{d}\right), \\
x_{i} x_{i+3}+\zeta_{i+3}\left(x_{1}, \ldots, \widehat{x_{i}}, \ldots, x_{d}\right), \\
x_{i} x_{i+4}+\zeta_{i+4}\left(x_{1}, \ldots, \widehat{x_{i}}, \ldots, x_{d}\right), \\
\vdots \\
x_{i} x_{i-2}+\zeta_{i-2}\left(x_{1}, \ldots, \widehat{x_{i}}, \ldots, x_{d}\right) .
\end{gathered}
$$

Hence, any linear first syzygy on $\left(I_{W_{d}}\right)_{2}$ must be a combination of Koszul syzygies on $x_{i+2}, x_{i+3}, \ldots, x_{i-2}$. Iterating this process for the term orders above shows there can be no linear first syzygies on $\left(I_{W_{d}}\right)_{2}$.

3D. Decomposition of $\mathbb{V}\left(\left\langle\left(\boldsymbol{I}_{W_{d}}\right)_{2}\right\rangle\right)$. We now prove that $\mathbb{V}\left(\left\langle\left(I_{W_{d}}\right)_{2}\right\rangle\right)=\mathscr{C} \cup W_{d}$. The results in Sections 4 and 5 are independent of this fact.

Lemma 3.10. For any $i, j$, and $k$, we have

$$
\left|\boldsymbol{n}_{i} \boldsymbol{n}_{j} \boldsymbol{n}_{k}\right|=\left|\boldsymbol{v}_{j} \boldsymbol{v}_{k} \boldsymbol{v}_{k+1}\right| \cdot\left|\boldsymbol{v}_{i} \boldsymbol{v}_{i+1} \boldsymbol{v}_{j+1}\right|-\left|\boldsymbol{v}_{j+1} \boldsymbol{v}_{k} \boldsymbol{v}_{k+1}\right| \cdot\left|\boldsymbol{v}_{i} \boldsymbol{v}_{i+1} \boldsymbol{v}_{j}\right|
$$

Proof. Apply the formulas $\boldsymbol{a} \times(\boldsymbol{b} \times \boldsymbol{c})=\boldsymbol{b}(\boldsymbol{a} \cdot \boldsymbol{c})-\boldsymbol{c}(\boldsymbol{a} \cdot \boldsymbol{b})$ and $|\boldsymbol{a b c}|=\boldsymbol{a} \times \boldsymbol{b} \cdot \boldsymbol{c}$ :

$$
\begin{aligned}
\left|\boldsymbol{n}_{i} \boldsymbol{n}_{j} \boldsymbol{n}_{k}\right| & =\boldsymbol{n}_{i} \times \boldsymbol{n}_{j} \cdot \boldsymbol{n}_{k}=\left(\boldsymbol{n}_{i} \times\left(\boldsymbol{v}_{j} \times \boldsymbol{v}_{j+1}\right)\right) \cdot \boldsymbol{n}_{k} \\
& =\left[\boldsymbol{v}_{j}\left(\boldsymbol{n}_{i} \cdot \boldsymbol{v}_{j+1}\right)-\boldsymbol{v}_{j+1}\left(\boldsymbol{n}_{i} \cdot \boldsymbol{v}_{j}\right)\right] \cdot \boldsymbol{n}_{k} \\
& =\left(\boldsymbol{v}_{j} \cdot \boldsymbol{n}_{k}\right)\left(\boldsymbol{n}_{i} \cdot \boldsymbol{v}_{j+1}\right)-\left(\boldsymbol{v}_{j+1} \cdot \boldsymbol{n}_{k}\right)\left(\boldsymbol{n}_{i} \cdot \boldsymbol{v}_{j}\right) \\
& =\left|\boldsymbol{v}_{j} \boldsymbol{v}_{k} \boldsymbol{v}_{k+1}\right| \cdot\left|\boldsymbol{v}_{i} \boldsymbol{v}_{i+1} \boldsymbol{v}_{j+1}\right|-\left|\boldsymbol{v}_{j+1} \boldsymbol{v}_{k} \boldsymbol{v}_{k+1}\right| \cdot\left|\boldsymbol{v}_{i} \boldsymbol{v}_{i+1} \boldsymbol{v}_{j}\right|
\end{aligned}
$$

Corollary 3.11. We have $\left|\boldsymbol{n}_{i} \boldsymbol{n}_{j} \boldsymbol{n}_{j+1}\right|=\alpha_{j+1}\left|\boldsymbol{v}_{i} \boldsymbol{v}_{i+1} \boldsymbol{v}_{j+1}\right|$.

Proof. This follows from Lemma 3.10 and the definition of $\alpha_{j+1}$.

Corollary 3.12. We have $\left|\boldsymbol{n}_{i-1} \boldsymbol{n}_{i} \boldsymbol{n}_{i+1}\right|=\alpha_{i} \alpha_{i+1}$.

Proof. This follows from Lemma 3.10 and the definition of $\alpha_{i}$ and $\alpha_{i+1}$.

Lemma 3.13. Let $\boldsymbol{x}=\left[x_{1}: \cdots: x_{d}\right] \in \mathbb{V}\left(\left\langle\left(I_{W_{d}}\right)_{2}\right\rangle\right) \backslash \mathscr{C}$. If $\tau(\boldsymbol{x})$ is a base point $p_{i j}=\boldsymbol{n}_{i} \times \boldsymbol{n}_{j}$, then $\boldsymbol{x}$ lies on the exceptional line $\hat{p}_{i j}$ over $p_{i j}$.

Proof. Since indices are cyclic, we assume that $i=1$. Thus, $\tau(\boldsymbol{x})=p_{1, j}=\boldsymbol{n}_{1} \times \boldsymbol{n}_{j}$ for some $j \notin\{d, 1,2\}$. The relation $Q_{1}(\boldsymbol{x})=\Lambda_{1} \cdot \tau(\boldsymbol{x})=\Lambda_{1} \cdot\left(\boldsymbol{n}_{1} \times \boldsymbol{n}_{j}\right)=0$ yields

$$
L(1):=x_{2} \boldsymbol{n}_{2} \cdot p_{1, j}-x_{1} \boldsymbol{n}_{d} \cdot p_{1, j}=0 .
$$

The relation $Q_{j}(\boldsymbol{x})=0$ implies

$$
L(j):=x_{j+1}\left|\boldsymbol{n}_{j+1} \boldsymbol{n}_{1} \boldsymbol{n}_{j}\right|-x_{j}\left|\boldsymbol{n}_{2} \boldsymbol{n}_{1} \boldsymbol{n}_{j}\right|=0 .
$$


Also,

$$
Q_{2}(\boldsymbol{x})=\left(x_{3} \boldsymbol{n}_{3}-x_{2} \boldsymbol{n}_{1}\right) \cdot \boldsymbol{n}_{1} \times \boldsymbol{n}_{j}=x_{3}\left|\boldsymbol{n}_{3} \boldsymbol{n}_{1} \boldsymbol{n}_{j}\right|=0,
$$

implying $x_{3}=0$ since $\left|\boldsymbol{n}_{3} \boldsymbol{n}_{1} \boldsymbol{n}_{j}\right| \neq 0$ if $j \neq 3$. Assume $x_{k}=0$ for $3 \leq k<j-1$. Note that

$$
Q_{k}(\boldsymbol{x})=\left(x_{k+1} \boldsymbol{n}_{k+1}-x_{k} \boldsymbol{n}_{k-1}\right) \cdot \boldsymbol{n}_{1} \times \boldsymbol{n}_{j}=x_{k+1}\left|\boldsymbol{n}_{k+1} \boldsymbol{n}_{1} \boldsymbol{n}_{j}\right|=0 ;
$$

hence, $x_{k+1}=0$ since $\left|\boldsymbol{n}_{k+1} \boldsymbol{n}_{1} \boldsymbol{n}_{j}\right| \neq 0$ and by induction $x_{k}=0$ for $3 \leq k \leq j-1$. An analogous argument shows that $x_{k}=0$ for $j+2 \leq k \leq d$. Hence, $\boldsymbol{x}$ lies on the line $\mathbb{V}\left(L(1), L(j), x_{k} \mid k \notin\{1,2, j, j+1\}\right)$, which is the exceptional line $\hat{p}_{1, j}$.

Theorem 3.14. The subset $\mathbb{V}\left(\left\langle\left(I_{W_{d}}\right)_{2}\right\rangle\right) \backslash \mathscr{C}$ is contained in $W_{d}$. It follows that the variety $\mathbb{V}\left(\left\langle\left(I_{W_{d}}\right)_{2}\right\rangle\right)$ has irreducible decomposition $W_{d} \cup \mathscr{C}$.

Proof. Let $\boldsymbol{x}=\left[x_{1}: \cdots: x_{d}\right] \in \mathbb{V}\left(\left\langle\left(I_{W_{d}}\right)_{2}\right\rangle\right) \backslash \mathscr{C}$. The Wachspress quadrics give the relations

$$
x_{r+1} \boldsymbol{n}_{r+1} \cdot \tau=x_{r} \boldsymbol{n}_{r-1} \cdot \tau
$$

for each $r=1, \ldots, d$. By Theorem 1.6, the adjoint is independent of triangulation,

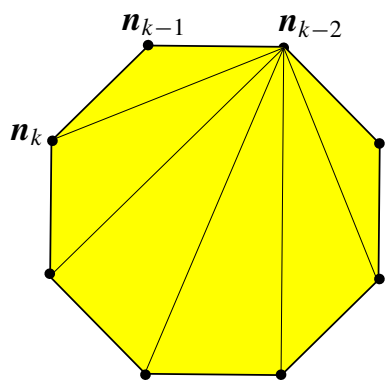

Figure 3. Triangulation used for adjoint.

so we use $\mathscr{A}$ to denote the adjoint, specifying the triangulation if necessary. We now show, for each $k \in\{1, \ldots, d\}, b_{k}(\tau(\boldsymbol{x}))=\mathscr{A}(\tau(\boldsymbol{x})) x_{k}$, where the triangulation above is used for the adjoint $\mathscr{A}$. It follows from the uniqueness of Wachspress coordinates that the denominator $\sum_{i=1}^{d} b_{i}$ of $\beta_{i}$ is the adjoint of $\boldsymbol{P}_{d}^{*}$, so it follows that

$$
w_{d}(\tau(\boldsymbol{x}))=\mathscr{A}(\tau(\boldsymbol{x})) \boldsymbol{x} .
$$

Provided $\mathscr{A}(\tau(\boldsymbol{x})) \neq 0$, the result follows since $w_{d}(\tau(\boldsymbol{x})) \in \mathbb{P}^{d-1}$ is a nonzero scalar multiple of $\boldsymbol{x}$; hence, $\boldsymbol{x}$ is in the image of the Wachspress map and thus lies on $W_{d}$. If $\boldsymbol{x} \in \mathbb{V}\left(\left\langle\left(I_{W_{d}}\right)_{2}\right\rangle\right) \backslash \mathscr{C}$ and $\mathscr{A}(\tau(\boldsymbol{x}))=0$, then by $(12) w_{d}(\tau(\boldsymbol{x}))=0$, and hence, $\tau(\boldsymbol{x})$ is a basepoint of $w_{d}$. Thus, $\tau(\boldsymbol{x})=\boldsymbol{n}_{i} \times \boldsymbol{n}_{j}$ for some diagonal pair $(i, j)$. By Lemma 3.13, 
$\boldsymbol{x}$ lies on an exceptional line and hence lies on $W_{d}$. To prove the claim, note that since all indices are cyclic it suffices to assume $k=3$. Let $\left|\boldsymbol{n}_{i} \boldsymbol{n}_{j} \boldsymbol{n}_{k}\right|=\left|\boldsymbol{n}_{i j k}\right|$ and

$$
\boldsymbol{n}_{i_{1}, \ldots, i_{m}} \cdot \tau:=\prod_{j=1}^{m}\left(\boldsymbol{n}_{i_{j}} \cdot \tau\right)
$$

This is the product of $m$ linear forms in $S$, and with this notation,

$$
b_{3}(\tau)=\boldsymbol{n}_{1,4,5, \ldots, d} \cdot \tau
$$

For each $r \in\{3, \ldots, d\}$, define

$\sigma_{r}:=\left(\boldsymbol{n}_{4, \ldots, r} \cdot \tau\right) \boldsymbol{n}_{1} \cdot\left[\sum_{i=3}^{r} \boldsymbol{v}_{i}\left(\boldsymbol{n}_{r+1, \ldots, d} \cdot \tau\right) x_{i}+\sum_{i=r+1}^{d} \boldsymbol{v}_{i}\left(\boldsymbol{n}_{r-1, \ldots, i-2} \cdot \tau\right)\left(\boldsymbol{n}_{i+1, \ldots, d} \cdot \tau\right) x_{r}\right]$,

where we set $\boldsymbol{n}_{i, \ldots, j} \cdot \tau=1$ if $j<i$. We show $x_{3} \mathscr{A}(\tau(\boldsymbol{x}))=\sigma_{3}=\sigma_{d}=b_{3}(\tau(\boldsymbol{x}))$. First, we show $\sigma_{3}=x_{3} \mathscr{A}(\tau)$ : to see this, note that

$$
x_{3} \mathscr{A}(\tau)=\left|\boldsymbol{n}_{123}\right|\left(\boldsymbol{n}_{4, \ldots, d} \cdot \tau\right) x_{3}+\sum_{i=4}^{d}\left|\boldsymbol{n}_{1, i-1, i}\right|\left(\boldsymbol{n}_{2, \ldots, i-2} \cdot \tau\right)\left(\boldsymbol{n}_{i+1, \ldots, d} \cdot \tau\right) x_{3},
$$

where we express the adjoint $\mathscr{A}$ using the triangulation in Figure 3. Applying the scalar triple product to $\left|\boldsymbol{n}_{123}\right|$ and $\left|\boldsymbol{n}_{1, i-1, i}\right|$ in the expression (13) yields

$$
\boldsymbol{n}_{1} \cdot\left(\boldsymbol{n}_{2} \times \boldsymbol{n}_{3}\right)\left(\boldsymbol{n}_{4, \ldots, d} \cdot \tau\right) x_{3}+\sum_{i=4}^{d} \boldsymbol{n}_{1} \cdot\left(\boldsymbol{n}_{i-1} \times \boldsymbol{n}_{i}\right)\left(\boldsymbol{n}_{2, \ldots, i-2} \cdot \tau\right)\left(\boldsymbol{n}_{i+1, \ldots, d} \cdot \tau\right) x_{3} .
$$

Factoring an $\boldsymbol{n}_{1}$ and noting that $\boldsymbol{n}_{i} \times \boldsymbol{n}_{i+1}=\boldsymbol{v}_{i+1}$, (14) becomes

$$
\boldsymbol{n}_{1} \cdot\left[\boldsymbol{v}_{3}\left(\boldsymbol{n}_{4, \ldots, d} \cdot \tau\right) x_{3}+\sum_{i=4}^{d} \boldsymbol{v}_{i}\left(\boldsymbol{n}_{2, \ldots, i-2} \cdot \tau\right)\left(\boldsymbol{n}_{i+1, \ldots, d} \cdot \tau\right) x_{3}\right]=\sigma_{3} .
$$

Now we show $\sigma_{d}=b_{3}(\tau)$. Since $\boldsymbol{n}_{d+1, \ldots, d} \cdot \tau=1$,

$$
\sigma_{d}=\left(\boldsymbol{n}_{4, \ldots, d} \cdot \tau\right) \boldsymbol{n}_{1} \cdot\left(\sum_{i+3}^{d} \boldsymbol{v}_{i}\left(\boldsymbol{n}_{d+1, \ldots, d} \cdot \tau\right) x_{i}\right)=\left(\boldsymbol{n}_{4, \ldots, d} \cdot \tau\right) \boldsymbol{n}_{1} \cdot\left(\sum_{i+3}^{d} \boldsymbol{v}_{i} x_{i}\right) .
$$

Observing that $\boldsymbol{n}_{1} \cdot \sum_{i=1}^{2} x_{i} \boldsymbol{v}_{i}=0$, we see that (15) is

$$
\left(\boldsymbol{n}_{4, \ldots, d} \cdot \tau\right)\left(\boldsymbol{n}_{1} \cdot \tau\right)=\boldsymbol{n}_{1,4, \ldots, d} \cdot \tau=b_{3}(\tau)
$$


We now claim that for $r \in\{3, \ldots, d-1\}$ we have $\sigma_{r}=\sigma_{r+1}$. Indeed,

$$
\begin{aligned}
\sigma_{r}=\left(\boldsymbol{n}_{4, \ldots, r} \cdot \tau\right) \boldsymbol{n}_{1} \cdot\left[\sum_{i=3}^{r} \boldsymbol{v}_{i}\left(\boldsymbol{n}_{r+1, \ldots, d} \cdot \tau\right) x_{i}\right. \\
\left.\quad+\sum_{i=r+1}^{d} \boldsymbol{v}_{i}\left(\boldsymbol{n}_{r, \ldots, i-2} \cdot \tau\right)\left(\boldsymbol{n}_{i+1, \ldots, d} \cdot \tau\right)\left(\boldsymbol{n}_{r-1} \cdot \tau\right) x_{r}\right] \\
=\left(\boldsymbol{n}_{4, \ldots, r} \cdot \tau\right) \boldsymbol{n}_{1} \cdot\left[\sum_{i=3}^{r} \boldsymbol{v}_{i}\left(\boldsymbol{n}_{r+1, \ldots, d} \cdot \tau\right) x_{i}\right. \\
\left.\quad+\sum_{i=r+1}^{d} \boldsymbol{v}_{i}\left(\boldsymbol{n}_{r, \ldots, i-2} \cdot \tau\right)\left(\boldsymbol{n}_{i+1, \ldots, d} \cdot \tau\right)\left(\boldsymbol{n}_{r+1} \cdot \tau\right) x_{r+1}\right],
\end{aligned}
$$

where we have applied (11) to the last term. Factoring out $\boldsymbol{n}_{r+1} \cdot \tau$ yields

$$
\left(\boldsymbol{n}_{4, \ldots, r+1} \cdot \tau\right) \boldsymbol{n}_{1} \cdot\left[\sum_{i=3}^{r} \boldsymbol{v}_{i}\left(\boldsymbol{n}_{r+2, \ldots, d} \cdot \tau\right) x_{i}+\sum_{i=r+1}^{d} \boldsymbol{v}_{i}\left(\boldsymbol{n}_{r, \ldots, i-2} \cdot \tau\right)\left(\boldsymbol{n}_{i+1, \ldots, d} \cdot \tau\right) x_{r+1}\right] .
$$

Lastly, since the expressions in both summations agree at the index $i=r+1$, we can shift the indices of summation,

$\left(\boldsymbol{n}_{4, \ldots, r+1} \cdot \tau\right) \boldsymbol{n}_{1} \cdot\left[\sum_{i=3}^{r+1} \boldsymbol{v}_{i}\left(\boldsymbol{n}_{r+2, \ldots, d} \cdot \tau\right) x_{i}+\sum_{i=r+2}^{d} \boldsymbol{v}_{i}\left(\boldsymbol{n}_{r, \ldots, i-2} \cdot \tau\right)\left(\boldsymbol{n}_{i+1, \ldots, d} \cdot \tau\right) x_{r+1}\right]$,

which is precisely $\sigma_{r+1}$, proving the claim. The claim shows that $\sigma_{3}=\sigma_{d}$; hence, (12) holds, and so $\boldsymbol{x}$ lies in $W_{d}$ if $\mathscr{A}(\tau(\boldsymbol{x})) \neq 0$.

\section{The Wachspress cubics}

Theorem 3.14 shows that the Wachspress quadrics do not suffice to cut out the Wachspress variety $W_{d}$. We now construct cubics, the Wachspress cubics, that lie in $I_{W_{d}}$ and do not arise from the Wachspress quadrics. These cubics are determinants of $3 \times 3$ matrices of linear forms. The key to showing that they are in $I_{W_{d}}$ is to write them as a difference of adjoints $\mathscr{A}_{T_{1}(C)}-\mathscr{A}_{T_{2}(C)}$, where $T_{1}(C)$ and $T_{2}(C)$ are two different triangulations of a subcone $C$ of the dual cone $\boldsymbol{P}_{d}^{*}$. By Theorem 1.6, the difference is zero, so the cubic is in $I_{W_{d}}$.

4A. Construction of Wachspress cubics. As in Lemma 3.6, let

$$
\Lambda_{r}=\frac{x_{r+1}}{\alpha_{r+1}} \boldsymbol{n}_{r+1}-\frac{x_{r}}{\alpha_{r}} \boldsymbol{n}_{r-1}
$$

Theorem 4.1. If $i \neq j \neq k \neq i$, then $w_{i, j, k}:=\left|\Lambda_{i}, \Lambda_{j}, \Lambda_{k}\right| \in I_{W_{d}}$.

Proof. We break the proof into two parts. First, suppose no pair of $(i, j, k)$ corresponds to an edge of $P_{d}$. We call such an $(i, j, k)$ a $T$-triple. A direct 
calculation shows that, if $(i, j, k)$ is a $T$-triple, then evaluating the monomial $x_{i} x_{j} x_{k}$ at Wachspress coordinates yields

$$
x_{i} x_{j} x_{k}\left(w_{d}\right)=b_{i} b_{j} b_{k}=A^{2} \prod_{m \in \gamma(i, j, k)} \ell_{m},
$$

where $\gamma(i, j, k)$ is as in Definition 3.4. Since there are no $T$-triples if $d<6$, we may assume $d \geq 6$. Changing variables by replacing $x_{i}$ with $x_{i} / \alpha_{i}$, we may ignore the constants $\alpha_{i}$. Using the definition of the $\Lambda$ 's, observe that

$$
\begin{aligned}
w_{i, j, k}= & \left|\boldsymbol{n}_{i+1} \boldsymbol{n}_{j+1} \boldsymbol{n}_{k+1}\right| x_{i+1} x_{j+1} x_{k+1}-\left|\boldsymbol{n}_{i+1} \boldsymbol{n}_{j+1} \boldsymbol{n}_{k-1}\right| x_{i+1} x_{j+1} x_{k} \\
& -\left|\boldsymbol{n}_{i+1} \boldsymbol{n}_{j-1} \boldsymbol{n}_{k+1}\right| x_{i+1} x_{j} x_{k+1}+\left|\boldsymbol{n}_{i+1} \boldsymbol{n}_{j-1} \boldsymbol{n}_{k-1}\right| x_{i+1} x_{j} x_{k} \\
& -\left|\boldsymbol{n}_{i-1} \boldsymbol{n}_{j+1} \boldsymbol{n}_{k+1}\right| x_{i} x_{j+1} x_{k+1}+\left|\boldsymbol{n}_{i-1} \boldsymbol{n}_{j+1} \boldsymbol{n}_{k-1}\right| x_{i} x_{j+1} x_{k} \\
& +\left|\boldsymbol{n}_{i-1} \boldsymbol{n}_{j-1} \boldsymbol{n}_{k+1}\right| x_{i} x_{j} x_{k+1}-\left|\boldsymbol{n}_{i-1} \boldsymbol{n}_{j-1} \boldsymbol{n}_{k-1}\right| x_{i} x_{j} x_{k} .
\end{aligned}
$$

There are several situations to consider, depending on various possibilities for interactions among the indices. Interactions may occur if $i+1=j-1$ or $j+1=k-1$ or $k+1=i-1$, so there are four cases:
1. All three hold.
2. Two hold.
3. One holds.
4. None hold.

Case 1. The indices $(i, j, k)$ satisfy Case 1 if and only if $d=6$. For $d=6$, there are only two $T$-triples: $(1,3,5)$ and $(2,4,6)$. We show that $w_{1,3,5}$ vanishes on Wachspress coordinates; the case of $w_{2,4,6}$ is similar. All but two of the determinants in Equation (17) vanish, leaving

$$
w_{1,3,5}=\left|\Lambda_{1}, \Lambda_{3}, \Lambda_{5}\right|=\left|\boldsymbol{n}_{2} \boldsymbol{n}_{4} \boldsymbol{n}_{6}\right| x_{2} x_{4} x_{6}-\left|\boldsymbol{n}_{6} \boldsymbol{n}_{2} \boldsymbol{n}_{4}\right| x_{1} x_{3} x_{5} .
$$

Notice that the coefficients are equal, and we conclude by showing that

$$
x_{1} x_{3} x_{5}-x_{2} x_{4} x_{6}
$$

vanishes on Wachspress coordinates. The monomials $x_{1} x_{3} x_{5}$ and $x_{2} x_{4} x_{6}$ evaluated at Wachspress coordinates are $b_{1} b_{3} b_{5}$ and $b_{2} b_{4} b_{6}$, respectively. Both of these are equal to $A^{2}$, so $x_{1} x_{3} x_{5}-x_{2} x_{4} x_{6}$ vanishes on Wachspress coordinates.

Case 2. We can assume without loss of generality $i+1 \neq j-1, j+1=k-1$, and $k+1=i-1$. Four coefficients vanish in (17), yielding

$$
\begin{aligned}
w_{i, j, k}= & \left|\boldsymbol{n}_{i+1} \boldsymbol{n}_{j+1} \boldsymbol{n}_{i-1}\right| x_{i+1} x_{j+1} x_{i-1} \\
& -\left|\boldsymbol{n}_{i+1} \boldsymbol{n}_{j-1} \boldsymbol{n}_{i-1}\right| x_{i+1} x_{j} x_{i-1} \\
& +\left|\boldsymbol{n}_{i+1} \boldsymbol{n}_{j-1} \boldsymbol{n}_{j+1}\right| x_{i+1} x_{j} x_{i-2} \\
& -\left|\boldsymbol{n}_{i-1} \boldsymbol{n}_{j-1} \boldsymbol{n}_{j+1}\right| x_{i} x_{j} x_{i-2} .
\end{aligned}
$$


Evaluating this at Wachspress coordinates yields

$$
\begin{array}{r}
w_{i, j, k} \circ w_{d}=\left|\boldsymbol{n}_{i+1} \boldsymbol{n}_{j+1} \boldsymbol{n}_{i-1}\right| \prod_{m \in \gamma(i+1, j+1, i-1)} \ell_{m}+\left|\boldsymbol{n}_{i+1} \boldsymbol{n}_{j-1} \boldsymbol{n}_{i-1}\right| \prod_{m \in \gamma(i+1, j, i-1)} \ell_{m} \\
-\left|\boldsymbol{n}_{i+1} \boldsymbol{n}_{j-1} \boldsymbol{n}_{j+1}\right| \prod_{m \in \gamma(i+1, j, i-1)} \ell_{m}-\left|\boldsymbol{n}_{i-1} \boldsymbol{n}_{j-1} \boldsymbol{n}_{j+1}\right| \prod_{m \in \gamma(i, j, i-1)} \ell_{m} \\
=A^{2}\left(\prod_{m \in \gamma(i-1, i+1, j+1, j)} \ell_{m}\right)\left(\left|\boldsymbol{n}_{i+1} \boldsymbol{n}_{j+1} \boldsymbol{n}_{i-1}\right| \ell_{j-1}-\left|\boldsymbol{n}_{i+1} \boldsymbol{n}_{j-1} \boldsymbol{n}_{i-1}\right| \ell_{j+1}\right. \\
\left.+\left|\boldsymbol{n}_{i+1} \boldsymbol{n}_{j-1} \boldsymbol{n}_{j+1}\right| \ell_{i-1}-\left|\boldsymbol{n}_{i-1} \boldsymbol{n}_{j-1} \boldsymbol{n}_{j+1}\right| \ell_{i+1}\right) \\
=A^{2}\left(\prod_{m \in \gamma(i-1, i+1, j+1, j)} \ell_{m}\right)\left[\left(\left|\boldsymbol{n}_{i+1} \boldsymbol{n}_{j+1} \boldsymbol{n}_{i-1}\right| \ell_{j-1}+\left|\boldsymbol{n}_{i-1} \boldsymbol{n}_{j+1} \boldsymbol{n}_{j-1}\right| \ell_{i+1}\right)\right. \\
\left.-\left(\left|\boldsymbol{n}_{i+1} \boldsymbol{n}_{j-1} \boldsymbol{n}_{i-1}\right| \ell_{j+1}+\left|\boldsymbol{n}_{i+1} \boldsymbol{n}_{j+1} \boldsymbol{n}_{j-1}\right| \ell_{i-1}\right)\right],
\end{array}
$$

where

$$
A=\prod_{i=1}^{d} \ell_{i} .
$$

The last factor is the difference of two adjoints with respect to the triangulations of the quadrilateral in Figure 4. The vanishing can be seen directly: write $\boldsymbol{n}_{1}, \ldots, \boldsymbol{n}_{4}$ for $\boldsymbol{n}_{i-1}, \boldsymbol{n}_{i+1}, \boldsymbol{n}_{j-1}$, and $\boldsymbol{n}_{j+1}$. Then the last factor is

$$
\left|\boldsymbol{n}_{2} \boldsymbol{n}_{3} \boldsymbol{n}_{4}\right| \ell_{1}-\left|\boldsymbol{n}_{1} \boldsymbol{n}_{3} \boldsymbol{n}_{4}\right| \ell_{2}+\left|\boldsymbol{n}_{1} \boldsymbol{n}_{2} \boldsymbol{n}_{4}\right| \ell_{3}-\left|\boldsymbol{n}_{1} \boldsymbol{n}_{2} \boldsymbol{n}_{3}\right| \ell_{4} .
$$

Applying $\frac{d}{d x}$ to this shows the $x$ coefficient is

$$
\left|n_{2} n_{3} n_{4}\right| n_{11}-\left|n_{1} n_{3} n_{4}\right| n_{21}+\left|n_{1} n_{2} n_{4}\right| n_{31}-\left|n_{1} n_{2} n_{3}\right| n_{41} .
$$

This is the determinant of the matrix of the $\boldsymbol{n}_{i}$ with a repeat row for the $x$ coordinates $\boldsymbol{n}_{i 1}$, so it vanishes. Reason similarly for the $y$ and $z$ coefficients.
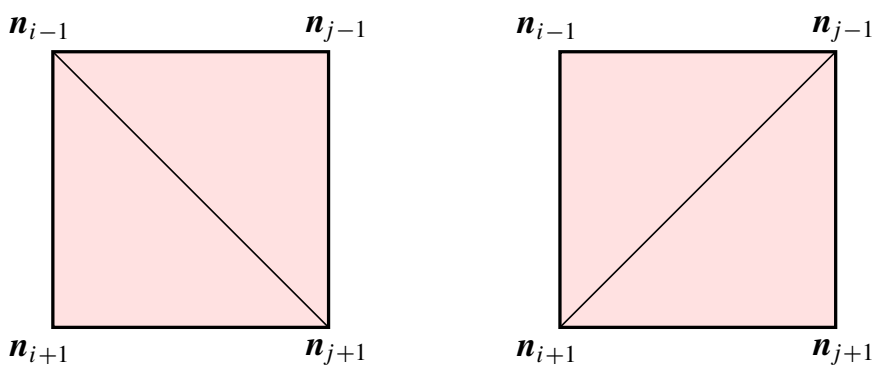

Figure 4. Case 2 triangulation. 

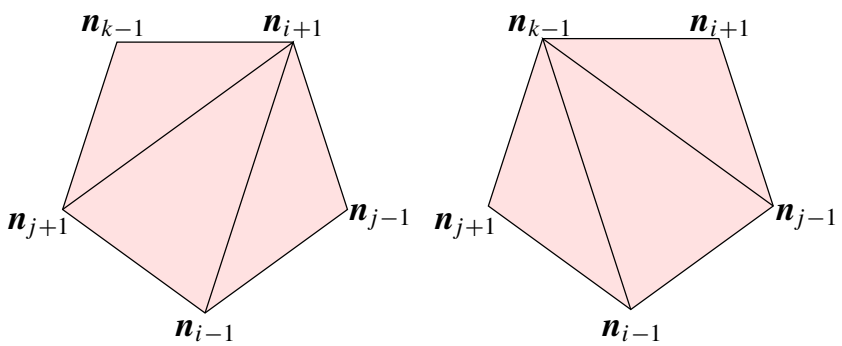

Figure 5. Case 3 triangulation.

Case 3. Assume without loss of generality $i+1 \neq j-1, j+1 \neq k-1$, and $k+1=i-1$. In this case, two coefficients vanish in (17), and after evaluating at Wachspress coordinates, we obtain

$$
\begin{gathered}
w_{i, j, k} \circ w_{d} \\
=\left|\boldsymbol{n}_{i+1} \boldsymbol{n}_{j+1} \boldsymbol{n}_{i-1}\right| \prod_{m \in \gamma} \prod_{i+1, j+1, k+1)} \ell_{m}-\left|\boldsymbol{n}_{i+1} \boldsymbol{n}_{j+1} \boldsymbol{n}_{k-1}\right| \prod_{m \in \gamma(i+1, j+1, k)} \ell_{m} \prod_{m \in \gamma(i+1, j, k+1)} \prod_{m}+\left|\boldsymbol{n}_{i+1} \boldsymbol{n}_{j-1} \boldsymbol{n}_{k-1}\right| \prod_{m \in \gamma} \ell_{m+1, j, k} \\
\quad-\left|\boldsymbol{n}_{i+1} \boldsymbol{n}_{j-1} \boldsymbol{n}_{i-1}\right| \prod_{m \in \gamma(i, j+1, k)} \ell_{m}-\left|\boldsymbol{n}_{i-1} \boldsymbol{n}_{j-1} \boldsymbol{n}_{k-1}\right| \prod_{m \in \gamma(i, j, k)} \ell_{m} \\
\left.+\left|\boldsymbol{n}_{i-1} \boldsymbol{n}_{j+1} \boldsymbol{n}_{k-1}\right| \prod_{i+1, j+1, k+1)} \ell_{m}\right)\left(\left|\boldsymbol{n}_{i+1} \boldsymbol{n}_{j+1} \boldsymbol{n}_{i-1}\right| \ell_{j-1} \ell_{k-1}-\left|\boldsymbol{n}_{i+1} \boldsymbol{n}_{j+1} \boldsymbol{n}_{k-1}\right| \ell_{i-1} \ell_{j-1}\right. \\
+\left|\boldsymbol{n}_{i+1} \boldsymbol{n}_{j-1} \boldsymbol{n}_{i-1}\right| \ell_{j+1} \ell_{k-1}+\left|\boldsymbol{n}_{i+1} \boldsymbol{n}_{j-1} \boldsymbol{n}_{k-1}\right| \ell_{j+1} \ell_{i-1} \\
\left.+\left|\boldsymbol{n}_{i-1} \boldsymbol{n}_{j+1} \boldsymbol{n}_{k-1}\right| \ell_{i+1} \ell_{j-1}-\left|\boldsymbol{n}_{i-1} \boldsymbol{n}_{j-1} \boldsymbol{n}_{k-1}\right| \ell_{i+1} \ell_{j+1}\right) .
\end{gathered}
$$

The last factor is the difference of adjoints with respect to the triangulations of the pentagon in Figure 5.

Case 4. In this case, evaluation at Wachspress coordinates yields

$$
\begin{aligned}
w_{i, j, k} \circ w_{d}=\left|\boldsymbol{n}_{i+1} \boldsymbol{n}_{j+1} \boldsymbol{n}_{k+1}\right| & \prod_{m \in \gamma(i+1, j+1, k+1)} \ell_{m}-\left|\boldsymbol{n}_{i+1} \boldsymbol{n}_{j+1} \boldsymbol{n}_{k-1}\right| \prod_{m \in \gamma(i+1, j+1, k)} \ell_{m} \\
& -\left|\boldsymbol{n}_{i+1} \boldsymbol{n}_{j-1} \boldsymbol{n}_{k+1}\right| \prod_{m \in \gamma(i+1, j, k+1)} \ell_{m}+\left|\boldsymbol{n}_{i+1} \boldsymbol{n}_{j-1} \boldsymbol{n}_{k-1}\right| \prod_{m \in \gamma(i+1, j, k)} \ell_{m} \\
& -\left|\boldsymbol{n}_{i-1} \boldsymbol{n}_{j+1} \boldsymbol{n}_{k+1}\right| \\
& +\left|\prod_{m \in \gamma(i, j+1, k+1)} \ell_{m}+\right| \boldsymbol{n}_{i-1} \boldsymbol{n}_{j+1} \boldsymbol{n}_{k-1} \mid \prod_{m \in \gamma(i, j+1, k)} \ell_{m} \\
& \prod_{j-1} \boldsymbol{n}_{k+1}\left|\prod_{m \in \gamma(i, j, k+1)} \ell_{m}-\right| \boldsymbol{n}_{i-1} \boldsymbol{n}_{j-1} \boldsymbol{n}_{k-1} \mid \prod_{m \in \gamma(i, j, k)} \ell_{m}
\end{aligned}
$$



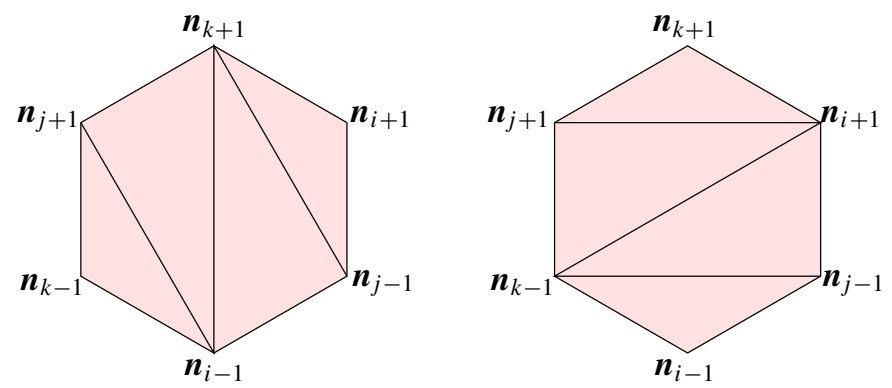

Figure 6. Case 4 triangulation.

$$
\begin{aligned}
=A^{2}\left(\prod_{\substack{m \in \gamma(i, j, k, i+1, j+1, k+1)}} \ell_{m}\right) \\
\times\left(\left|\boldsymbol{n}_{i+1} \boldsymbol{n}_{j+1} \boldsymbol{n}_{k+1}\right| \ell_{i-1} \ell_{j-1} \ell_{k-1}-\left|\boldsymbol{n}_{i+1} \boldsymbol{n}_{j+1} \boldsymbol{n}_{k-1}\right| \ell_{i-1} \ell_{j-1} \ell_{k+1}\right. \\
\quad-\left|\boldsymbol{n}_{i+1} \boldsymbol{n}_{j-1} \boldsymbol{n}_{k+1}\right| \ell_{i-1} \ell_{j+1} \ell_{k-1}+\left|\boldsymbol{n}_{i+1} \boldsymbol{n}_{j-1} \boldsymbol{n}_{k-1}\right| \ell_{i-1} \ell_{j+1} \ell_{k+1} \\
\quad-\left|\boldsymbol{n}_{i-1} \boldsymbol{n}_{j+1} \boldsymbol{n}_{k+1}\right| \ell_{i+1} \ell_{j-1} \ell_{k-1}+\left|\boldsymbol{n}_{i-1} \boldsymbol{n}_{j+1} \boldsymbol{n}_{k-1}\right| \ell_{j+1} \ell_{i-1} \ell_{k+1} \\
\left.\quad+\left|\boldsymbol{n}_{i-1} \boldsymbol{n}_{j-1} \boldsymbol{n}_{k+1}\right| \ell_{i+1} \ell_{j+1} \ell_{k-1}-\left|\boldsymbol{n}_{i-1} \boldsymbol{n}_{j-1} \boldsymbol{n}_{k-1}\right| \ell_{i+1} \ell_{j+1} \ell_{k+1}\right) .
\end{aligned}
$$

The last factor is the difference of adjoints expressed using the triangulations of the hexagon in Figure 6. This completes the analysis when $(i, j, k)$ is a $T$-triple.

Next, we consider the situation when $(i, j, k)$ contains a pair of consecutive indices. Suppose first that there are exactly two consecutive vertices; without loss of generality, we assume the indices are $(2,3, i)$ with $i>4$. We have

$$
\begin{aligned}
w_{2,3, i}:=\left|\Lambda_{2} \Lambda_{3} \Lambda_{i}\right|= & \left|\boldsymbol{n}_{2} \boldsymbol{n}_{4} \boldsymbol{n}_{i+1}\right| x_{3} x_{4} x_{i+1}-\left|\boldsymbol{n}_{3} \boldsymbol{n}_{4} \boldsymbol{n}_{i-1}\right| x_{3} x_{4} x_{i} \\
& -\left|\boldsymbol{n}_{3} \boldsymbol{n}_{2} \boldsymbol{n}_{i+1}\right| x_{3} x_{3} x_{i+1}+\left|\boldsymbol{n}_{3} \boldsymbol{n}_{2} \boldsymbol{n}_{i-1}\right| x_{3} x_{3} x_{i} \\
& -\left|\boldsymbol{n}_{1} \boldsymbol{n}_{4} \boldsymbol{n}_{i+1}\right| x_{2} x_{4} x_{i+1}+\left|\boldsymbol{n}_{1} \boldsymbol{n}_{4} \boldsymbol{n}_{i-1}\right| x_{2} x_{4} x_{i} \\
& +\left|\boldsymbol{n}_{1} \boldsymbol{n}_{2} \boldsymbol{n}_{i+1}\right| x_{2} x_{3} x_{i+1}-\left|\boldsymbol{n}_{1} \boldsymbol{n}_{2} \boldsymbol{n}_{i-1}\right| x_{2} x_{3} x_{i} .
\end{aligned}
$$

We show that $w_{2,3, i} \circ w_{d}$ is a multiple of the difference between two expressions of the adjoint polynomial of a polygon with respect to two different triangulations. After evaluation at $w_{d}$, each monomial has a common factor of $A \prod_{j \neq 2,3} \ell_{j}$. Thus, we can express

$$
\frac{w_{2,3, i}\left(w_{d}\right)}{A \prod_{j \neq 2,3} \ell_{j}}
$$



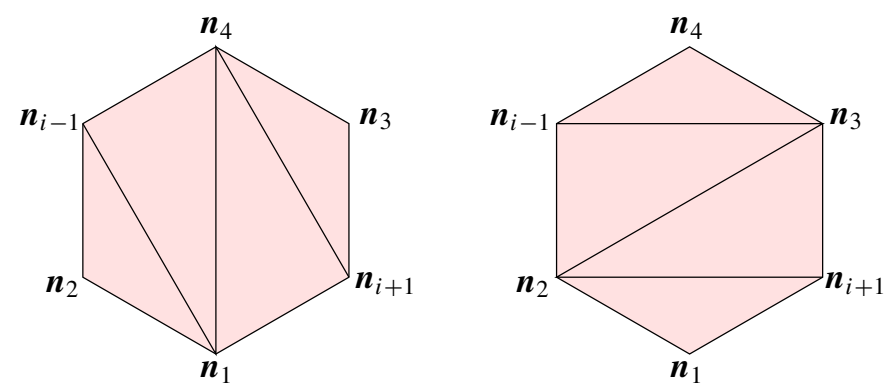

Figure 7. Triangulations for the non- $T$-triples.

as

$$
\begin{aligned}
& \frac{w_{2,3, i}\left(w_{d}\right)}{A \prod_{j \neq 2,3} \ell_{j}}=\left|\boldsymbol{n}_{2} \boldsymbol{n}_{4} \boldsymbol{n}_{i+1}\right| \prod_{j \neq 3,4, i+1} \ell_{j}-\left|\boldsymbol{n}_{3} \boldsymbol{n}_{4} \boldsymbol{n}_{i-1}\right| \prod_{j \neq 3,4, i-1} \ell_{j} \\
& -\left|\boldsymbol{n}_{3} \boldsymbol{n}_{2} \boldsymbol{n}_{i+1}\right| \prod_{j \neq 2,3, i+1} \ell_{j}+\left|\boldsymbol{n}_{3} \boldsymbol{n}_{2} \boldsymbol{n}_{i-1}\right| \prod_{j \neq 2,3, i-1} \ell_{j} \\
& -\left|\boldsymbol{n}_{1} \boldsymbol{n}_{4} \boldsymbol{n}_{i+1}\right| \prod_{j \neq 1,4, i+1} \ell_{j}+\left|\boldsymbol{n}_{1} \boldsymbol{n}_{4} \boldsymbol{n}_{i-1}\right| \prod_{j \neq 1,4, i-1} \ell_{j} \\
& +\left|\boldsymbol{n}_{1} \boldsymbol{n}_{2} \boldsymbol{n}_{i+1}\right| \prod_{j \neq 1,2, i+1} \ell_{j}-\left|\boldsymbol{n}_{1} \boldsymbol{n}_{2} \boldsymbol{n}_{i-1}\right| \prod_{j \neq 1,2, i-1} \ell_{j} \\
& =\left(\prod_{j \in \gamma(2,4, i, i+1)} \ell_{j}\right)\left(\left|\boldsymbol{n}_{2} \boldsymbol{n}_{4} \boldsymbol{n}_{i+1}\right| \ell_{1} \ell_{3} \ell_{i-1}-\left|\boldsymbol{n}_{3} \boldsymbol{n}_{4} \boldsymbol{n}_{i-1}\right| \ell_{1} \ell_{2} \ell_{i+1}\right. \\
& -\left|\boldsymbol{n}_{3} \boldsymbol{n}_{2} \boldsymbol{n}_{i+1}\right| \ell_{1} \ell_{4} \ell_{i-1}+\left|\boldsymbol{n}_{3} \boldsymbol{n}_{2} \boldsymbol{n}_{i-1}\right| \ell_{1} \ell_{4} \ell_{i+1} \\
& -\left|\boldsymbol{n}_{1} \boldsymbol{n}_{4} \boldsymbol{n}_{i+1}\right| \ell_{2} \ell_{3} \ell_{i-1}+\left|\boldsymbol{n}_{1} \boldsymbol{n}_{4} \boldsymbol{n}_{i-1}\right| \ell_{2} \ell_{3} \ell_{i+1} \\
& \left.+\left|\boldsymbol{n}_{1} \boldsymbol{n}_{2} \boldsymbol{n}_{i+1}\right| \ell_{3} \ell_{4} \ell_{i-1}-\left|\boldsymbol{n}_{1} \boldsymbol{n}_{2} \boldsymbol{n}_{i-1}\right| \ell_{3} \ell_{4} \ell_{i+1}\right) \text {. }
\end{aligned}
$$

The factor in parentheses is the difference of the adjoints computed with respect to the triangulations of the polygon in Figure 7.

Finally, for the case where the three vertices are consecutive, assume without loss of generality the triple is $(2,3,4)$, and proceed as above. In this case, the triangulations that arise are those that appear in Figure 5.

Definition 4.2. $I(d)$ is the ideal generated by the Wachspress quadrics appearing in Corollary 3.8 and the Wachspress cubics appearing in Theorem 4.1.

\section{Gröbner basis, Stanley-Reisner ring, and free resolution}

In this section, we determine the initial ideal of $I(d)$ in graded lex order and prove $I(d)=I_{W_{d}}$. First, we present some preliminaries. 
5A. Simplicial complexes and combinatorial commutative algebra. An abstract $n$-simplex is a set consisting of all subsets of an $(n+1)$-element ground set. Typically a simplex is viewed as a geometric object; for example, a 2-simplex on the set $\{a, b, c\}$ can be visualized as a triangle with the subset $\{a, b, c\}$ corresponding to the whole triangle, $\{a, b\}$ an edge, and $\{a\}$ a vertex. For this reason, elements of the ground set are called the vertices.

Definition 5.1 [Ziegler 1995]. A simplicial complex $\Delta$ on a vertex set $V$ is a collection of subsets $\sigma$ of $V$ such that, if $\sigma \in \Delta$ and $\tau \subset \sigma$, then $\tau \in \Delta$. If $|\sigma|=i+1$, then $\sigma$ is called an $i$-face. Let $f_{i}(\Delta)$ denote the number of $i$-faces of $\Delta$, and define $\operatorname{dim}(\Delta)=\max \left\{i \mid f_{i}(\Delta) \neq 0\right\}$. If $\operatorname{dim}(\Delta)=n-1$, we define $f_{\Delta}(t)=\sum_{i=0}^{n} f_{i-1} t^{n-i}$. The ordered list of coefficients of $f_{\Delta}(t)$ is the $f$-vector of $\Delta$, and the coefficients of $h_{\Delta}(t):=f_{\Delta}(t-1)$ are the $h$-vector of $\Delta$.

Example 5.2. Consider the 1-skeleton of a tetrahedron with vertices $x_{1}, x_{2}, x_{3}, x_{4}$, as in the figure.

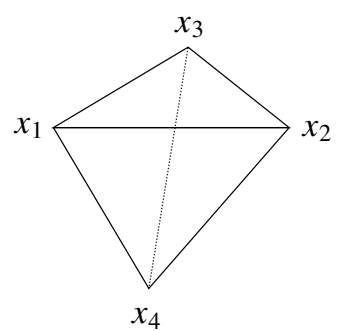

The corresponding simplicial complex $\Delta$ consists of all vertices and edges, so $\Delta=\left\{\varnothing,\left\{x_{i}\right\},\left\{x_{i}, x_{j}\right\} \mid 1 \leq i \leq 4\right.$ and $\left.i<j \leq 4\right\}$. Thus, $f(\Delta)=(1,4,6)$ and $h(\Delta)=(1,2,3)$; the empty face gives $f_{-1}(\Delta)=1$.

A simplicial complex $\Delta$ can be used to define a commutative ring, known as the Stanley-Reisner ring. This construction allows us to use tools of commutative algebra to prove results about the topology or combinatorics of $\Delta$.

Definition 5.3. Let $\Delta$ be a simplicial complex on vertices $\left\{x_{1}, \ldots, x_{n}\right\}$. The StanleyReisner ideal $I_{\Delta}$ is

$$
\left.I_{\Delta}=\left\langle x_{i_{1}} \cdots x_{i_{j}}\right|\left\{x_{i_{1}}, \ldots, x_{i_{j}}\right\} \text { is not a face of } \Delta\right\rangle \subseteq \mathbb{K}\left[x_{1}, \ldots, x_{n}\right],
$$

and the Stanley-Reisner ring is $\mathbb{K}\left[x_{1}, \ldots, x_{k}\right] / I_{\Delta}$.

In Example 5.2, since $\Delta$ has no 2-faces,

$$
I_{\Delta}=\left\langle x_{1} x_{2} x_{3}, x_{1} x_{2} x_{4}, x_{1} x_{3} x_{4}, x_{2} x_{3} x_{4}\right\rangle=\bigcap_{1 \leq i<j \leq 4}\left\langle x_{i}, x_{j}\right\rangle .
$$

Definition 5.4. A prime ideal $P$ is associated to a graded $S$-module $N$ if $P$ is the annihilator of some $n \in N$, and $\operatorname{Ass}(N)$ is the set of all associated primes of $N$. 
Definition 5.5. Let $\operatorname{codim}(N)=\min \{\operatorname{codim}(P) \mid P \in \operatorname{Ass}(N)\}$ for a finitely generated graded $S$-module $N$. The projective $\operatorname{dimension} \operatorname{pdim}(N)$ is the length of a minimal free resolution of $N ; N$ is Cohen-Macaulay if $\operatorname{codim}(N)=\operatorname{pdim}(N)$. $S / I$ is arithmetically Cohen-Macaulay if it is Cohen-Macaulay as an $S$-module.

\section{B. Application to Wachspress surfaces.}

Definition 5.6. Define $I_{\Gamma}(d) \subseteq \mathbb{K}\left[x_{1}, \ldots, x_{d}\right]$ as

$$
I_{\Gamma}(d)=\left\langle x_{1} x_{3}, \ldots, x_{1} x_{d-1}\right\rangle+K_{2, d-1},
$$

where $K_{2, d-1}$ consists of all square-free cubic monomials in $x_{2}, \ldots, x_{d-1}$.

Theorem 5.7. The quotient $S / I_{\Gamma}(d)$ is arithmetically Cohen-Macaulay, of Castelnuovo-Mumford regularity two, and has Hilbert series

$$
\operatorname{HS}\left(S / I_{\Gamma}(d), t\right)=\frac{1+(d-3) t+\left(\begin{array}{c}
d-3 \\
2
\end{array}\right) t^{2}}{(1-t)^{3}} .
$$

Proof. The ideal $I_{\Gamma}(d)$ is the Stanley-Reisner ideal of a one-dimensional simplicial complex $\Gamma$ consisting of a complete graph on vertices $\left\{x_{2}, \ldots, x_{d-1}\right\}$ with a single additional edge $\overline{x_{1} x_{2}}$ attached. All connected graphs are shellable, so since shellable implies Cohen-Macaulay (see [Miller and Sturmfels 2005]), $S / I_{\Gamma}(d)$ is CohenMacaulay. Since $I_{\Gamma}(d)$ contains no terms involving $x_{d}$, if $S^{\prime}=\mathbb{K}\left[x_{1}, \ldots, x_{d-1}\right]$, then

$$
S / I_{\Gamma}(d) \simeq S^{\prime} / I_{\Gamma}(d) \otimes \mathbb{K}\left[x_{d}\right] .
$$

The Hilbert series of a Stanley-Reisner ring has numerator equal to the $h$-vector of the associated simplicial complex (see [Schenck 2003]), which in this case is a graph on $d-1$ vertices with $\left(\begin{array}{c}d-2 \\ 2\end{array}\right)+1$ edges. Converting $f(\Gamma)=\left(1, d-1,\left(\begin{array}{c}d-2 \\ 2\end{array}\right)+1\right)$ to $h(\Gamma)$ yields the Hilbert series of $S^{\prime} / I_{\Gamma}(d)$. The Hilbert series of a graph has denominator $(1-t)^{2}$, and tensoring with $\mathbb{K}\left[x_{d}\right]$ contributes a factor of $1 /(1-t)$, yielding the result.

Theorem 5.8. In graded lex order, in $_{\prec} I(d)=I_{\Gamma}(d)$.

Proof. First, note that

$$
I_{\Gamma}(d) \subseteq \text { in }_{\prec} I(d),
$$

which follows from Corollary 3.8 and Theorem 4.1, combined with the observation that, in graded lex order, in $\left(\left|\Lambda_{i} \Lambda_{j} \Lambda_{k}\right|\right)=x_{i} x_{j} x_{k}$ if $i<j<k$ as long as $k \neq d$. Since $I(d) \subseteq I_{W_{d}}$, there is a surjection

$$
S / I(d) \rightarrow S / I_{W_{d}}
$$

hence, $\operatorname{HP}(S / I(d), t) \geq \operatorname{HP}\left(S / I_{W_{d}}, t\right)$. Since

$$
\operatorname{HP}(S / I(d), t)=\operatorname{HP}\left(S / \operatorname{in}_{\prec} I(d), t\right)
$$


and

$$
I_{\Gamma}(d) \subseteq \operatorname{in}_{\prec} I(d)
$$

we have

$$
\operatorname{HP}\left(S / I_{\Gamma}(d), t\right) \geq \operatorname{HP}\left(S / \operatorname{in}_{\prec} I(d), t\right)=\operatorname{HP}(S / I(d), t) \geq \operatorname{HP}\left(S / I_{W_{d}}, t\right) .
$$

The Hilbert polynomial $\operatorname{HP}\left(S / I_{W_{d}}, t\right)$ is given by Equation (5). The Hilbert series of $S / I_{\Gamma}(d)$ is given by Theorem 5.7, from which we can extract the Hilbert polynomial:

$$
\operatorname{HP}\left(S / I_{\Gamma}(d), t\right)=\left(\begin{array}{c}
d-3 \\
2
\end{array}\right)\left(\begin{array}{l}
t \\
2
\end{array}\right)+(d-3)\left(\begin{array}{c}
t+1 \\
2
\end{array}\right)+\left(\begin{array}{c}
t+2 \\
2
\end{array}\right),
$$

and a check shows this agrees with Equation (5). Since $I_{\Gamma}(d) \subseteq$ in $_{\prec} I(d)$, equality of the Hilbert polynomials implies that in high degree (i.e., up to saturation)

$$
I_{\Gamma}(d)=\operatorname{in}_{\prec} I(d) \quad \text { and } \quad I(d)=I_{W_{d}} .
$$

Consider the short exact sequence

$$
0 \rightarrow \operatorname{in}_{\prec} I(d) / I_{\Gamma}(d) \rightarrow S / I_{\Gamma}(d) \rightarrow S / \text { in }_{\prec} I(d) \rightarrow 0 .
$$

By Lemma 3.6 of [Eisenbud 1995],

$$
\operatorname{Ass}\left(\operatorname{in}_{\prec} I(d) / I_{\Gamma}(d)\right) \subseteq \operatorname{Ass}\left(S / I_{\Gamma}(d)\right) .
$$

Since $\operatorname{HP}\left(S / I_{\Gamma}(d), t\right)=\operatorname{HP}\left(S /\right.$ in $\left._{\prec} I(d), t\right)$, the module in $I_{\prec}(d) / I_{\Gamma}(d)$ must vanish in high degree so is supported at $\mathfrak{m}$, which is of codimension $d$. But $I_{\Gamma}(d)$ is a radical ideal supported in codimension $d-3$, so it follows from Equation (19) that in $_{\prec} I(d) / I_{\Gamma}(d)$ must vanish.

Corollary 5.9. The ideal $I(d)$ is the ideal of the image of

$$
X_{d} \rightarrow \mathbb{P}\left(H^{0}\left(D_{d-2}\right)\right) .
$$

In particular, $I(d)=I_{W_{d}}$, and $S / I(d)$ is arithmetically Cohen-Macaulay.

Proof. By the results of Sections 2 and 3, $I(d) \subseteq I_{W_{d}}$, and the proof of Theorem 5.8 showed that they are equal up to saturation. Hence, $I_{W_{d}} / I(d)$ is supported at $\mathfrak{m}$. Consider the short exact sequence

$$
0 \rightarrow I_{W_{d}} / I(d) \rightarrow S / I(d) \rightarrow S /\left(I_{W_{d}}\right) \rightarrow 0 .
$$

Since $S / I_{\Gamma}(d)=S /$ in $_{\prec} I(d)$ is arithmetically Cohen-Macaulay of codimension $d-3$, by uppersemicontinuity [Herzog 2005], so is $S / I(d)$, so $I_{W_{d}} / I(d)=0$.

Corollary 5.10. The quotient $S / I_{W_{d}}$ has regularity 2.

Proof. Since $S / I(d)$ is Cohen-Macaulay, reducing modulo a linear regular sequence of length 3 yields an Artinian ring with the same regularity, which is equal to the socle degree [Eisenbud 2005]. By Theorems 5.7 and 5.8, this is 2, so the regularity of $S / I_{W_{d}}$ is 2 . 
Theorem 5.11. The nonzero graded Betti numbers of the minimal free resolution of $S / I(d)$ are given by $b_{12}=d-3$ and for $i \geq 3$ by

$$
b_{i-2, i}=\left(\begin{array}{c}
d-3 \\
i
\end{array}\right)-(d-3)\left(\begin{array}{c}
d-3 \\
i-1
\end{array}\right)+\left(\begin{array}{c}
d-3 \\
2
\end{array}\right)\left(\begin{array}{c}
d-3 \\
i-2
\end{array}\right) .
$$

Proof. By Corollary 5.10, there are only two rows in the Betti table of $S / I(d)$. By Corollary 3.9, the top row is empty, save for the quadratic generators at the first step. Thus, the entire Betti diagram may be obtained from the Hilbert series, which is given in Theorem 5.7, and the result follows.

We are at work on generalizing the results here to higher dimensions.

\section{Acknowledgments}

Computations were performed using Macaulay2, by Grayson and Stillman, available at http://www.math.uiuc.edu/Macaulay2/. A script is available at http://www.math. uiuc.edu/ schenck/Wpress.m2/. Our collaboration began during a SIAM conference on applied algebraic geometry, and we thank the organizers of that conference, Dan Bates and Frank Sottile. We thank Frank Sottile and Greg Smith for useful comments. Irving also thanks his thesis advisor, Frank Sottile, for introducing him to Wachspress varieties and providing guidance during the dissertation process.

\section{References}

[Buchsbaum and Eisenbud 1973] D. A. Buchsbaum and D. Eisenbud, "What makes a complex exact?”, J. Algebra 25 (1973), 259-268. MR 47 \#3369 Zbl 0264.13007

[Cox et al. 2011] D. A. Cox, J. B. Little, and H. K. Schenck, Toric varieties, Graduate Studies in Mathematics 124, American Mathematical Society, Providence, RI, 2011. MR 2012g:14094 Zbl 1223.14001

[Davis and Geramita 1988] E. D. Davis and A. V. Geramita, "Birational morphisms to $\mathbf{P}^{2}$ : an ideal-theoretic perspective”, Math. Ann. 279:3 (1988), 435-448. MR 89a:14014 Zbl 0657.14003

[Eisenbud 1995] D. Eisenbud, Commutative algebra, with a view toward algebraic geometry, Graduate Texts in Mathematics 150, Springer, New York, 1995. MR 97a:13001 Zbl 0819.13001

[Eisenbud 2005] D. Eisenbud, The geometry of syzygies: a second course in commutative algebra and algebraic geometry, Graduate Texts in Mathematics 229, Springer, New York, 2005. MR 2005h:13021 Zbl 1066.14001

[Garcia-Puente and Sottile 2010] L. D. Garcia-Puente and F. Sottile, "Linear precision for parametric patches”, Adv. Comput. Math. 33:2 (2010), 191-214. MR 2011f:65030 Zbl 1193.65018

[Harbourne 2002] B. Harbourne, "Problems and progress: a survey on fat points in $\mathbf{P}^{2}$ ", pp. 85-132 in Zero-dimensional schemes and applications (Naples, 2000), edited by A. V. Geramita, Queen's Papers in Pure and Appl. Math. 123, Queen's Univ., Kingston, ON, 2002. MR 2003f:13032 Zbl 1052.14052

[Hartshorne 1977] R. Hartshorne, Algebraic geometry, Graduate Texts in Mathematics 52, Springer, New York, 1977. MR 57 \#3116 Zbl 0367.14001 
[Herzog 2005] J. Herzog, "Finite free resolutions", pp. 118-144 in Computational commutative and non-commutative algebraic geometry, edited by S. Cojocaru et al., NATO Sci. Ser. III Comput. Syst. Sci. 196, IOS, Amsterdam, 2005. MR 2007a:13014 Zbl 1105.13012

[Miller and Sturmfels 2005] E. Miller and B. Sturmfels, Combinatorial commutative algebra, Graduate Texts in Mathematics 227, Springer, New York, 2005. MR 2006d:13001 Zbl 1066.13001

[Möbius 1827] A. F. Möbius, Der barycentrische Calcul: ein neues Hülfsmittel zur analytischen Behandlung der Geometrie, Johann Ambrosius Barth, Leipzig, 1827.

[Schenck 2003] H. Schenck, Computational algebraic geometry, London Mathematical Society Student Texts 58, Cambridge University Press, 2003. MR 2004k:13001 Zbl 1046.14034

[Wachspress 1975] E. L. Wachspress, A rational finite element basis, Mathematics in Science and Engineering 114, Academic Press, New York, 1975. MR 54 \#14403 Zbl 0322.65001

[Warren 1996] J. Warren, "Barycentric coordinates for convex polytopes", Adv. Comput. Math. 6:2 (1996), 97-108. MR 97m:52026 Zbl 0873.52013

[Warren 2003] J. Warren, "On the uniqueness of barycentric coordinates", pp. 93-99 in Topics in algebraic geometry and geometric modeling, edited by R. Goldman and R. Krasauskas, Contemp. Math. 334, Amer. Math. Soc., Providence, RI, 2003. MR 2005b:52022 Zbl 1043.52009

[Ziegler 1995] G. M. Ziegler, Lectures on polytopes, Graduate Texts in Mathematics 152, Springer, New York, 1995. MR 96a:52011 Zbl 0823.52002

Communicated by Craig Huneke

Received 2013-01-06 Revised 2013-04-07 Accepted 2013-05-27

cfirving@scu.edu

schenck@math.uiuc.edu
Department of Mathematics and Computer Science, Santa Clara University, 500 El Camino Real, Santa Clara, CA 95053, United States

Department of Mathematics, University of Illinois at Urbana-Champaign, 1409 West Green Street, Urbana, IL 61801, United States 


\section{Algebra \& Number Theory}

msp.org/ant

\section{EDITORS}

MANAGING EDITOR

Bjorn Poonen

Massachusetts Institute of Technology

Cambridge, USA

\author{
EDITORIAL BOARD CHAIR \\ David Eisenbud \\ University of California \\ Berkeley, USA
}

\section{BOARD OF EDITORS}

Georgia Benkart

Dave Benson

Richard E. Borcherds

John H. Coates

J-L. Colliot-Thélène

Brian D. Conrad

Hélène Esnault

Hubert Flenner

Edward Frenkel

Andrew Granville

Joseph Gubeladze

Roger Heath-Brown

Ehud Hrushovski

Craig Huneke

Mikhail Kapranov

Yujiro Kawamata

János Kollár

Yuri Manin

Barry Mazur

Philippe Michel
University of Wisconsin, Madison, USA

University of Aberdeen, Scotland

University of California, Berkeley, USA

University of Cambridge, UK

CNRS, Université Paris-Sud, France

University of Michigan, USA

Freie Universität Berlin, Germany

Ruhr-Universität, Germany

University of California, Berkeley, USA

Université de Montréal, Canada

San Francisco State University, USA

Oxford University, UK

Hebrew University, Israel

University of Virginia, USA

Yale University, USA

University of Tokyo, Japan

Princeton University, USA

Northwestern University, USA

Harvard University, USA

École Polytechnique Fédérale de Lausanne
Susan Montgomery

Shigefumi Mori

Raman Parimala

Jonathan Pila

Victor Reiner

Karl Rubin

Peter Sarnak

Joseph H. Silverman

Michael Singer

Vasudevan Srinivas

J. Toby Stafford

Bernd Sturmfels

Richard Taylor

Ravi Vakil

Michel van den Bergh

Marie-France Vignéras

Kei-Ichi Watanabe

Efim Zelmanov

Shou-Wu Zhang
University of Southern California, USA

RIMS, Kyoto University, Japan

Emory University, USA

University of Oxford, UK

University of Minnesota, USA

University of California, Irvine, USA

Princeton University, USA

Brown University, USA

North Carolina State University, USA

Tata Inst. of Fund. Research, India

University of Michigan, USA

University of California, Berkeley, USA

Harvard University, USA

Stanford University, USA

Hasselt University, Belgium

Université Paris VII, France

Nihon University, Japan

University of California, San Diego, USA

Princeton University, USA

PRODUCTION

production@msp.org

Silvio Levy, Scientific Editor

See inside back cover or msp.org/ant for submission instructions.

The subscription price for 2014 is US $\$ 225 /$ year for the electronic version, and $\$ 400 /$ year $(+\$ 55$, if shipping outside the US) for print and electronic. Subscriptions, requests for back issues and changes of subscribers address should be sent to MSP.

Algebra \& Number Theory (ISSN 1944-7833 electronic, 1937-0652 printed) at Mathematical Sciences Publishers, 798 Evans Hall \#3840, c/o University of California, Berkeley, CA 94720-3840 is published continuously online. Periodical rate postage paid at Berkeley, CA 94704, and additional mailing offices.

ANT peer review and production are managed by EditFLOW ${ }^{\circledR}$ from Mathematical Sciences Publishers.

\section{PUBLISHED BY}

- mathematical sciences publishers

nonprofit scientific publishing

http://msp.org/

(C) 2014 Mathematical Sciences Publishers 


\section{Algebra \& Number Theory}

Volume $8 \quad$ No. $2 \quad 2014$

Large self-injective rings and the generating hypothesis

LEIGH SHEPPERSON and NEIL STRICKLAND

On lower ramification subgroups and canonical subgroups

303

SHIN HATTORI

Wild models of curves

DINO LORENZINI

Geometry of Wachspress surfaces

COREY IRVING and HAL SCHENCK

Daniel Goldstein, Robert M. GuRAlnick, Mark L. Lewis, AleXander Moretó, Gabriel Navarro and Pham HuU TieP

The homotopy category of injectives

AMNON NEEMAN

Essential dimension of spinor and Clifford groups

Vladimir Chernousov and AleXANDER MERKurJeV

On Deligne's category $\underline{\operatorname{Rep}}^{a b}\left(S_{d}\right)$

JONATHAN COMES and VICTOR OSTRIK

Algebraicity of the zeta function associated to a matrix over a free group algebra

CHRISTIAN KASSEL and Christophe REUTENAUER 